\title{
Structural basis of tubulin detyrosination by the vasohibin-SVBP enzyme complex
}

Na Wang ${ }^{1^{*}}$, Christophe Bosc ${ }^{2 *}$, Sung Ryul Choi ${ }^{3^{*}}$, Benoit Boulan ${ }^{2}$, Leticia Peris ${ }^{2}$, Natacha Olieric ${ }^{3}$, Hongyu Bao ${ }^{1}$, Fatma Krichen ${ }^{2}$, Liu Chen ${ }^{1}$, Annie Andrieux ${ }^{2}$, Vincent Olieric ${ }^{4}$, Marie-Jo Moutin ${ }^{2}$, Michel O. Steinmetz ${ }^{3,5}$, Hongda Huang ${ }^{1}$

${ }^{1}$ Department of Biology, Southern University of Science and Technology, Shenzhen, China

${ }^{2}$ Univ. Grenoble Alpes, Inserm, U1216, CNRS, CEA, Grenoble Institut Neurosciences, 38000 Grenoble, France

${ }^{3}$ Laboratory of Biomolecular Research, Division of Biology and Chemistry, Paul Scherrer Institut, $\mathrm{CH}$ 5232 Villigen PSI, Switzerland

${ }^{4}$ Laboratory for Macromolecules and Bioimaging, Photon Science Division, Paul Scherrer Institut, $\mathrm{CH}-$ 5232 Villigen PSI, Switzerland

${ }^{5}$ University of Basel, Biozentrum, $\mathrm{CH}-4056$ Basel, Switzerland

${ }^{1}$ Department of Biology, Southern University of Science and Technology, Shenzhen, China 2Univ. Grenoble Alpes, Inserm U1216, Grenoble Institut des Neurosciences, 38000 Grenoble, France ${ }^{3}$ Laboratory of Biomolecular Research, Division of Biology and Chemistry, Paul Scherrer Institut, $\mathrm{CH}$ 5232 Villigen PSI, Switzerland

${ }^{4}$ Univ. Grenoble Alpes, Inserm U1216, CEA-IRIG, Grenoble Institut des Neurosciences 38000 Grenoble, France

${ }^{5}$ Laboratory of for Macromolecules and Bioimaging, Photon Science Division, Paul Scherrer Institut, CH-5232 Villigen PSI, Switzerland

${ }^{6}$ Univ. Grenoble Alpes, Inserm U1216, CNRS, Grenoble Institut des Neurosciences 38000 Grenoble, France

${ }^{7}$ University of Basel, Biozentrum, $\mathrm{CH}-4056$ Basel, Switzerland

*These authors contributed equally to the work

Corresponding authors: moutinm@univ-grenoble-alpes.fr, michel.steinmetz@psi.ch, huanghd@sustc.edu.cn 


\section{Abstract}

Vasohibins are tubulin tyrosine carboxypeptidases that are important for neuron physiology. We solved crystal structures of human vasohibin 1 and 2 in complex with small vasohibin-binding protein (SVBP) in the absence and presence of different inhibitors and a C-terminal $\alpha$-tubulin peptide. In combination with functional data, we propose that SVBP acts as an activator of vasohibins. An extended groove and a distinctive surface residue patch of vasohibins define the specificity determinants for recognizing and cleaving the C-terminal tyrosine of $\alpha$-tubulin and for binding microtubules, respectively. The vasohibin-SVBP interaction and the ability of the enzyme complex to associate with microtubules regulate axon specification of neurons. Our results define the structural basis of tubulin detyrosination by vasohibins and show the relevance of this process for neuronal development. They further offer a unique platform for developing drugs against human conditions with abnormal tubulin tyrosination levels including cancer, heart defects and possibly brain disorders. 


\section{Introduction}

The evolutionarily conserved tubulin detyrosination-tyrosination cycle is important for mitosis, muscle mechanotransduction and neuron development ${ }^{1-11}$. It is thus not surprising that abnormal tubulin tyrosination levels are associated with severe human pathologies including cancer, heart failures, cardiomyopathies, and brain dysfunctions ${ }^{2,3,12-15}$ (reviewed $\mathrm{in}^{16}$ ). The tubulin detyrosinationtyrosination cycle is characterized by the enzymatic removal and re-addition of a gene-encoded tyrosine residue at the $\mathrm{C}$-terminus of $\alpha$-tubulin (reviewed $\mathrm{in}^{17,18}$ ). The enzyme that adds back the tyrosine residue, tubulin tyrosine ligase (TTL), has been studied in detail ${ }^{19-22}$. Notably, TTL-null mice die right after birth due to disorganized neuronal networks, highlighting the vital role of the tubulin detyrosination-tyrosination cycle for brain development ${ }^{12}$. An enzyme that removes the tyrosine, tubulin tyrosine carboxypeptidase (TCP), has been discovered only recently and identified as a stable heterodimeric complex formed between vasohibin 1 or vasohibin 2 and small vasohibin-binding protein (SVBP) 23,24 .

On the molecular level, the tubulin detyrosination-tyrosination cycle controls the activity of key cytoskeletal proteins, including the microtubule dynamics regulator CLIP-170 $7,9,25$, the dynein motor regulator $\mathrm{p} 150^{\text {glued } 9}$, the kinesin microtubule depolymerase $\mathrm{MCAK}^{26}$, and specific kinesin motors controlling intracellular neuron trafficking and development ${ }^{8,10,27,28}$. In the cases of CLIP-170 and p150 glued, their cytoskeleton-associated protein-glycine-rich (CAP-Gly) domains specifically recognize C-terminal EEY/F motifs such as found at the C-terminus of $\alpha$-tubulin (reviewed $\mathrm{in}^{29}$ ).

With the recent discovery of vasohibins as major TCPs in brain ${ }^{23,24}$, representative molecular players that "erase", "write" and "read" the tubulin tyrosine signal emerging from the tubulin detyrosination-tyrosination cycle have been defined. However, while the molecular mechanisms of the writer (TTL) and the readers (CAP-Gly proteins, kinesins) are well understood, little is known about the mechanisms of the erasers (TCPs) that actually initiate the cycle. Here, we sought to fill this gap in knowledge by establishing the structure-function relationship of tubulin detyrosination by the vasohibin-SVBP enzyme complex. 


\section{Results}

\section{Overall structure of vasohibin-SVBP enzyme complex}

As a first step, we solved the crystal structures of full length human vasohibin 2 (V2) and of the predicted structured core domains of V2 (V2c) and vasohibin 1 (V1c) in complex with SVBP to high resolution (Table 1-3). In both V2-SVBP, V2c-SVBP and V1c-SVBP almost the same amino acids of the proteins were visible in the respective complex structures and all three structures superimposed well with one another, showing a high degree of structural conservation (Supplementary Fig. 1a,b). Tubulin detyrosination assays in HEK293T cells revealed that under the experimental conditions tested V2c-SVBP is as active as V2-SVBP (Supplementary Figs $1 \mathrm{c}$ and 2a), suggesting that the disordered $\mathrm{N}$ - and C-terminal flanking regions of $\mathrm{V} 2$ are marginally important for the enzymatic mechanism of vasohibins. We thus focused on the V2c-SVBP system for the following structural analyses.

The V2c structure can be subdivided into an $\mathrm{N}$ - and a C-terminal domain (Fig. 1a). The helical Nterminal domain (ND) wraps around a long helix formed by the C-terminal segment of SVBP to form a three-helix bundle. The C-terminal domain of V2c (CD) is formed by a central antiparallel $\beta$-sheet that is flanked by two helical subdomains and comprises the predicted catalytic residues of vasohibins ${ }^{30}$. The ND and CD are packed together via SVBP that is sandwiched between both domains.

A structure-based similarity search using Dali ${ }^{31}$ revealed a similarity of the V2c-SVBP structure with members of the transglutaminase-like cysteine protease superfamily including large adhesion protein G $\left(\right.$ LapG $;{ }^{32}$ ), coagulation factor XIII ${ }^{33}$ and arylamine $\mathrm{N}$-acetyltransferase 1 (NAT1; ${ }^{34}$ ) (Z-scores between 5 and 8), consistent with a previous bioinformatics study ${ }^{30}$. As shown in Fig. 1b-e, the catalytic core formed by helices $\alpha 3, \alpha 4$ and $\alpha 5$ and beta strands $\beta 1, \beta 2$, and $\beta 3$ of V2c as well as the geometry of the catalytic residue triad involving the side chains of $\mathrm{C} 158$ and $\mathrm{H} 193$ and the backbone carbonyl group of L215 (see next section for a more detailed analysis) are similar to the ones of LapG, coagulation factor XIII and NAT1. However, besides the similar catalytic core architecture, the structures of the non-equivalent parts of the four enzymes are different and form distinctive channels and pockets for binding their respective cognate substrates.

Although carboxypeptidase A (CPA) can readily detyrosinate $\alpha$-tubulin in vitro ${ }^{26}$, our Dali search did not reveal any similarity between the V2c-SVBP and CPA ${ }^{35}$ structures. In fact, no structural similarity of V2c-SVBP to any canonical carboxypeptidases was found. Together, these results define the vasohibin-SVBP family as carboxypeptidases with a transglutaminase-like fold. 


\section{Vasohibin-SVBP active site architecture and molecular basis of inhibition}

Inspection of the surface of the V2c-SVBP structure revealed an extended and deep groove that is shaped and surrounded by several conserved V2c lysine and arginine residues (Fig. 2a, Supplementary Figs 3a,b and Supplementary Note). A predominantly hydrophobic pocket containing the catalytic residues of $\mathrm{V} 2 \mathrm{c}$ is located at the bottom of this groove. A bioinformatics study predicted a non-canonical catalytic residue triad Cys-His-Ser to form the active site of vasohibins ${ }^{30}$. However, our analysis of the V2c-SVBP structure revealed that this triad actually involves the side chains of C158 and H193 and the backbone carbonyl of L215 of V2c (denoted the Cys-His-(carbonyl)Leu triad; Fig. 2b). Notably, these three residues are strictly conserved among vasohibins in all the species that we examined (Supplementary Note and data not shown). The observed and characteristic Cys-His(carbonyl)Leu triad configuration thus represents a conserved and intrinsic feature of the vasohibinSVBP family of carboxypeptidases, which has been described in a few other cysteine proteases like, for example, SpeB and caspases ${ }^{36,37}$.

To test the relevance of the side chains of the catalytic triad in V2, we performed tubulin detyrosination assays in vitro with purified proteins and in HEK293T cells. As shown in Fig. 2c and Supplementary Figs $2 b$ and $4 a-j$, mutating $L 215$ to alanine did reduce the initial enzymatic rate of the V2 mutant ten-fold in vitro compared to wild type (30 nM enzyme concentration). However, the same mutation did not affect the detyrosinated tubulin levels in cells (Fig. 2c). In contrast, the activity of V2 was essentially abrogated both in vitro and in cells when mutating individually C158 or H193 to alanines. These results are consistent with the Cys-His-(carbonyl)Leu triad configuration observed in the V2c-SVBP structure and suggest that the side chains of C158 and H193 are critical for the enzymatic mechanism while the one of Leu215 is less critical.

To obtain further insights into the active site architecture of V2c and the determinants that recognize the C-terminal tyrosine of $\alpha$-tubulin, we solved a crystal structure of V2c-SVBP in complex with the tyrosine residue-based inhibitor of vasohibin, epoY ${ }^{24}$ (V2c-SVBP-epoY; Table 2 and Supplementary Fig. $3 c, d)$. The V2c-SVBP-epoY complex structure reveals that the tyrosine moiety of epoY is indeed bound to the identified hydrophobic pocket of V2c-SVBP (hereafter referred to as the tyrosinebinding pocket; Fig. 2a,d). As predicted previously ${ }^{24}$, the epoxy C3 atom of epoY is covalently attached to the catalytic residue C158 of V2c (Fig. 2e and Supplementary Fig. 3e). Furthermore, several residue side chains forming the tyrosine-binding pocket of $\mathrm{V} 2 \mathrm{c}$ undergo some rearrangements upon epoY binding to accommodate the compound (Supplementary Fig. 3f). 
Four distinct sets of highly conserved residues of V2c interact with four groups of the tyrosine moiety of epoY (Fig. 2e): (i) Y123, S210, R211 and H193 of V2c establish hydrogen bonds with the carboxylate group of epoY, corroborating vasohibin-SVBP as carboxypeptidase; (ii) F191, R211 and $\mathrm{H} 241$ of V2c interact with the phenyl ring of epoY through $\pi$ interactions, and F186, R212, L215 and Y236 further stabilize the phenyl ring through hydrophobic interactions; (iii) R212 and H241 of V2c interact with the hydroxyl group of epoY via water-mediated and direct hydrogen bonds, respectively; (iv) the backbone carbonyl of $\mathrm{H} 192$ of V2c forms a hydrogen bond with the amide group of epoY. Tubulin detyrosination assays in HEK293T cells with selected alanine point mutants revealed that F186, F191, R212, Y236, and H241 are not critical for the enzymatic activity of V2 (Supplementary Fig. 1c and 2a). Under the same experimental conditions, the Y123A and S210A V2 mutants produced $\sim 35 \%$ less detyrosinated tubulin compared to wild type, suggesting a relevance of these residues for the enzyme's activity (Fig. 2c and Supplementary Fig. 2b). In the case of the R211A mutant, detyrosination was almost abolished in cells suggesting that R211, like the catalytic triad residues C158 and H193, is essential for activity. In vitro tubulin detyrosination assays with purified proteins confirmed that mutating each of these three latter residues strongly affected the activity of V2-SVBP as testified by decreased initial enzymatic rates of at least fifty-fold (130 nM enzyme concentration; Supplementary Fig. 4j).

Collectively, these results suggest that the V2c-SVBP-epoY structure mimics well the interaction of V2c's catalytic site with a C-terminal tyrosine residue. Interestingly, R211 of V2 and the corresponding R222 of V1 are mutated to histidine and glutamine, respectively, in human patients suffering from different types of carcinomas (https://cancer.sanger.ac.uk/cosmic). Similar to the alanine substitutions, these patient mutations severely impaired the enzymatic activities of V1 and V2 in HEK293T cells (Fig. 2f and Supplementary Fig. 2c).

To establish a molecular basis of vasohibin inhibition with small molecules, we solved in addition to V2c-SVBP-epoY the high resolution crystal structure of V2c-SVBP in complex with the generic protease inhibitor TPCK (V2c-SVBP-TPCK; Table 2, Fig. 2g and Supplementary Fig. 3g,h), which inhibits vasohibins with good potency ${ }^{24}$. Similarly to epoY, TPCK is also bound to the tyrosine-binding pocket of V2c with the $\mathrm{C} 1$ atom of the compound covalently attached to the catalytic residue C158 (Fig. 2h). The tosyl and phenyl moieties of TPCK are stacked on each other and wedged into the tyrosinebinding pocket of V2c, which is wider compared to the one seen in V2-SVBP-epoY complex structure due to the more bulky nature of TPCK (Supplementary Fig. 3f,i). However, most V2c residues that contact the tyrosine moiety of epoY also interact with the tosyl and phenyl moieties of TPCK (Fig. 2e,h). The comparison of the V2c-SVBP-epoY and V2c-SVBP-TPCK structures further revealed that the tyrosine and the tosyl and phenyl moieties occupy a similar space in the tyrosine-binding pocket and 
are oriented in a very similar manner. Notably, epoY and TPCK inhibit vasohibins with very good potency while the other previously tested protease inhibitors TLCK, E-64 and parthenolide show only modest inhibitory activity ${ }^{24}$. Inspection of our crystal structures and the chemical structures of all the five compounds (Supplementary Fig. 3c,g,j-I) suggest that the presence of carboxylate group and tyrosine or phenylalanine ring mimics in a small molecule should allow achieving potency and likely also specificity towards vasohibins.

As mentioned in the previous section, the structures of V2c-SVBP and CPA are unrelated. Nevertheless, how they bind C-terminal tyrosine residues of substrates is somewhat similar: the carboxylate group is recognized through hydrogen bonding interactions and the aromatic ring of the tyrosine is bound to a predominantly hydrophobic pocket (Fig. 2e and Supplementary Fig. $3 \mathrm{~m}$ ). While CPA does not discriminate between C-terminal aromatic or bulky aliphatic amino acids $(F, Y, W, L, I)$ and displays a large substrate specificity ${ }^{35}$, vasohibin-SVBP are specific to C-terminal tyrosine or phenylalanine residues that are preceded by negatively charged residues ${ }^{23,24}$.

These results establish the molecular determinants of vasohibin-SVBP enzymes for recognizing and cleaving the C-terminal tyrosine of $\alpha$-tubulin. They further offer a structural basis to understand vasohibin-related patient mutations and to rationally design specific vasohibin inhibitors.

\section{Interaction of vasohibin-SVBP with a C-terminal $\alpha$-tubulin peptide}

We sought to test whether the extended, positively charged groove of V2c (Fig. 2a) interacts with the negatively charged C-terminal tail of $\alpha$-tubulin (Supplementary Fig. 5a). Crystallization attempts of V2c-SVBP in the presence of $\alpha$-tubulin tail peptides of different lengths did not produce a co-complex structure in our hands. We thus used the $\alpha$-tubulin derived mutant peptide 431DYEEVGVDSVEGEGEEEGECY-451 (maTail, with E450 mutated to cysteine) for crystallization, which was designed to form a disulfide bridge with the active site residue C158 of V2c based on our V2cSVBP-epoY complex structure. As SVBP also contains a solvent exposed cysteine at residue position 58, we mutated it to serine to avoid formation of a non-specific disulfide bridge between SVBP and the maTail peptide. Using this strategy, we solved the crystal structure of the V2c-SVBP-maTail complex (Table 2, Fig. 3a and Supplementary Fig. 5b).

Residues 447-450 of maTail could be modeled in a straightforward manner in the V2c-SVBP-maTail structure. The carboxylate group of the terminal Y451 is less well resolved most likely due to the unnatural covalent bond formed between C450 and C158 of maTail and V2c, respectively, which 
seems to hinder $\mathrm{Y} 451$ to achieve its optimal binding pose. Nevertheless, the resulting best position and orientation of $\mathrm{Y} 451$ in the tyrosine-binding pocket is similar to the one observed for the tyrosine moiety of epoY in the V2c-SVBP-epoY complex structure (Supplementary Fig. 5c,d), which validates our approach. A prominent salt-bridge interaction is established between the side chains of the highly conserved residues K135 of the positively charged groove of V2c and E447 of maTail. Additional contacts are formed between the backbone amide of $\mathrm{H} 192$ and the side chain of E449, and between the side chain of K157 with the backbone carbonyl of G448 of V2c and $\alpha$-tubulin, respectively. These interactions are expected to help anchoring the $\alpha$-tubulin tail in a manner to allow for optimal interaction of the C-terminal tyrosine of $\alpha$-tubulin with the active site and tyrosinebinding pocket residues of V2c-SVBP, thus facilitating the enzymatic reaction.

In line with this hypothesis, mutating individually K135 or K157 of V2 to alanine produced 80 and $\sim 75 \%$, respectively, less detyrosinated microtubules in HEK293T cells under the experimental conditions used (Fig. 3c and Supplementary Fig. 2d). Mutating the nearby residue R134 of V2, which does not form a direct contact with maTail, affected also the activity of the enzyme although to a lesser extent ( $\sim 0 \%$ less detyrosinated microtubules). Interestingly, the corresponding residue in V1, $\mathrm{K} 145$, is mutated to glutamate in a human patient suffering from ovarian carcinoma. Similarly to R134 of V2, mutation of this residue to alanine or glutamate partially reduced the enzymatic activity of V1 in HEK293T cells ( $\sim 0 \%$ and $60 \%$ less detyrosinated microtubules, respectively; Fig. $2 \mathrm{f}$ and Supplementary Fig. 2c). Together, these results define the molecular determinants of vasohibin-SVBP, which specifically recognize the negatively charged C-terminal tail of $\alpha$-tubulin.

\section{Interaction between vasohibin and SVBP}

Inspection of the V2c-SVBP structure revealed major hydrophobic contacts that are established between highly conserved residues stemming from the two helices a1 and a2 and the loop L2 of V2c's ND, and highly conserved residues of the SVBP helix (Fig. 4a and Supplementary Fig. 5e). Additional hydrogen bonding and hydrophobic interactions with SVBP are established by main chain and side chain interactions stemming from residues of loops L1 and L3 of V2c's ND, and loops L4, L5 and L9 of V2c's CD, which further stabilize the V2c-SVBP interaction (Fig. 4b,c). Interestingly, the SVBP helix is in direct contact with loops L4 and L9, two structural elements that are involved in shaping the tyrosine-binding pocket of V2c (Fig. 4c). This observation indicates a possible allosteric crosstalk between SVBP and the active site of vasohibins. 
To test the role of conserved residues for the vasohibin-SVBP interaction, we sought to perform mutagenesis in combination with pull down experiments. In our hands, it was not possible to express well-behaved vasohibin proteins in bacterial cells in the absence of SVBP. However, stable and soluble vasohibin proteins could be readily expressed when $\mathrm{N}$-terminally fused to maltose-binding protein (MBP). Isothermal titration calorimetry (ITC) revealed a tight 1:1 interaction between purified MBP-V2C and SVBP (Supplementary Fig. 5f), demonstrating that our MBP-fusion approach indeed produces well folded and functional vasohibin samples. The obtained equilibrium dissociation constant, $\mathrm{Kd}$, of $18 \pm 3 \mathrm{nM}$ is in reasonable agreement with values obtained previously by plasmon resonance for the V2-SVBP and V1-SVBP interactions ${ }^{38}$.

Simultaneous mutation of the interacting and conserved residues W63 and W67 of helix a 1 or L154 and P155 of loop L5 of V2c to glutamates, or deletion of the ND (V2c $\triangle N D$ ) all disrupted complex formation between purified MBP-V2 or MBP-V2c with GST-SVBP in pull down experiments (Fig. 4d and Supplementary Fig. 5g). A reduction in complex formation was also obtained for MBP-V2c and GST-SVBP double mutants in which the conserved residues Q35 and R36, I39 and Y40, or L42 and N43 of SVBP were pairwise mutated to alanines, while the double mutants V45A M46A and E50A F54A showed no effects (Fig. 4e). Co-expression of the same set of interface disrupting mutations in HEK293T cells using V2-eGFP and SVBP-myc-Flag constructs significantly reduced V2 protein levels (Fig. 4f,g and Supplementary Figs 2e,f), in line with the idea that the vasohibin-SVBP interaction is important for vasohibin stability ${ }^{23,38}$. Concurrently, a strongly reduced detyrosination activity was observed in these cells: under the experimental conditions used, we observed a reduction of detyrosinated tubulin by $60 \%$ for the three SVBP double mutants, concomitant with an almost similar reduction of soluble V2 protein production for these mutants (Fig. 4f,g and Supplementary Fig. $2 e, f)$. In vitro tubulin detyrosination assays with purified MBP-V2c, MBP-V2c $\triangle N D, M B P-V 2 c$ W63E W67E, MBP-V2C L154E P155E in the absence or presence of wild type SVBP or the double mutant SVBP I39A Y40A in turn revealed that the interaction between V2 and SVBP is important for the enzymatic activity (Fig. 4h,i). Together with our observation that the SVBP helix contacts the active site of V2c (Fig. 4c), these results suggest that SVBP, besides its chaperone function, acts as an activator of vasohibins.

\section{Functional analysis of vasohibin-SVBP}

Vasohibin-SVBP is more active on microtubules compared to free tubulin ${ }^{23,24}$.To test whether specific residues are involved in binding specific determinants of the negatively-charged microtubule lattice, we inspected the surface of the V2c-SVBP structure and identified a patch formed by the four highly 
conserved basic residues R137, K147, R151, and H257 of V2c, which are located at a distance from both the tyrosine-binding pocket and $\alpha$-tubulin tail-binding region (Fig. 5a). Mutating these four residues in V2 simultaneously to alanine (V2 4A) did not affect the fold of the protein (Supplementary Fig. 6) but did lead to an almost complete loss of detyrosination activity in HEK293T cells (Fig. 5b and Supplementary Fig. 2g). Interestingly, while wild type V2-SVBP showed a strong association with microtubules when expressed in NIH3T3 fibroblasts, V2 4A and SVBP colocalized only weakly with microtubules (Fig. 5c,d).

To test whether the decreased activity and localization of the V2 4A to microtubules in cells are consequences of a reduction of the direct binding of this mutant to microtubules, we performed pelleting assays with purified components. As shown in Fig. 5e, V2-SVBP binds specifically to microtubules with a stoichiometry of 1:1 V2-SVBP:tubulin dimer and with a Kd of $0.16 \pm 0.04 \mu \mathrm{M}$, consistent with the formation of a tight complex. Mutating Cys158 of V2 to alanine did not result in a reduced binding of the V2 C158A-SVBP mutant enzyme complex, suggesting that an intact catalytic triade is not important for the interaction (Fig. 5f). Interestingly, in contrast to what was found in cells V2 4A-SVBP did not show a significant reduced binding to microtubules neither. Similarly, in vitro experiments revealed no reduction in enzymatic activity for the V2 4A-SVBP mutant (Fig. 5g). We wondered whether the presence of the $\mathrm{C}$-terminal $\alpha$-tubulin tail that interacts with the positively charged groove of $\mathrm{V} 2 \mathrm{c}$ might interfere in vitro with the effect of the $4 \mathrm{~A}$ mutations at the high V2:tubulin ratios used in our experiments. To test this possibility, we performed limited proteolysis experiments with subtilisin that removes the disordered C-terminal tails of both $\alpha$ - and $\beta$-tubulin ${ }^{39}$. As shown in Fig. 5h, cleavage of the tubulin tails significantly reduced the binding of V2 4A-SVBP to microtubules, compared to wild type and V2 C158A-SVBP, which bound intact and cleaved microtubules similarly. This result indicates that the surface patch of V2 plays a role for recognizing the structured part of tubulin dimers in microtubules in vitro.

To test whether the interaction between vasohibin and SVBP and whether the association of the enzyme complex with microtubules are important for neuron development, in particular axon differentiation ${ }^{24}$, we performed experiments in neurons. As shown in Fig. 6a and Supplementary Fig. 7a, wild type human SVBP (hSVBP) was able to rescue the levels of detyrosinated tubulin and the delay of differentiation (revealed by a significant decrease of stage III neurons) of mouse hippocampal neurons knocked down for mouse SVBP (sh mSVBP). In contrast, the hSVBP mutant in which the 139 and Y40 residues were simultaneously mutated to alanines was unable to do so.

We then studied the phenotypic effects of boosting expression of wild type or mutated versions of V2 in hippocampal neurons. To this end, we tested wild type V2, a catalytic dead version of V2 in 
which C158 was mutated to alanine, or V2 4A that poorly associates with microtubules and very weakly detyrosinates tubulin in cycling cells. In contrast to the overexpression of hSVBP when mSVBP is down regulated, overexpression of wild type $\mathrm{V} 2$ induced premature axonal differentiation in neurons (Fig. 6a,b). Remarkably, it also induced a significant three-fold increase of abnormal neurons containing two axons (Fig. 6c,d), which is reminiscent to the phenotype observed in TTL null neurons ${ }^{12}$. As SVBP plays a key role for the structure and activity of vasohibin, its cellular levels might have been increased in our overexpression experiments to drive the observed phenotype. In contrast to wild type V2, the mutants V2 C158A and V2 4A displayed the same phenotype as the eGFP control.

Taken together, these results indicate that a distinct surface patch of positively charged residues on vasohibin-SVBP is important for the association of the enzyme with microtubules and its activity in cells. They further suggest that the vasohibin-SVBP interaction as well as the ability of the enzyme to associate with microtubules is involved in axon development. Our finding that the conserved and positively charged surface patch of V2 plays a less pronounced role in vitro indicates that in cells additional factors that, for example, bind to the microtubule shaft might modulate the interaction and activity of the enzyme complex. 


\section{Discussion}

Vasohibins are ubiquitously expressed proteins that were originally associated with angiogenesis (reviewed $\mathrm{in}^{40}$ ). However, very recently they were identified as major TCPs of the evolutionary conserved tubulin detyrosination-tyrosination cycle $^{23,24}$. In collaboration with TTL, TCPs generate distinct sub-populations of detyrosinated and tyrosinated microtubules in cells, and prime for additional $\alpha$-tubulin C-terminal tail modifications including the formation of $\Delta 2$ - and $\Delta 3$-tubulin ${ }^{41}$.

In this study, we found that the structure of vasohibins, while similar to members of the transglutaminase-like cysteine protease superfamily, contains a characteristic active site configuration defined by a non-canonical catalytic residue triad ${ }^{30}$. Distinctive structural elements on the surface of the vasohibin-SVBP enzyme complex and encoded by highly conserved residues across vasohibin 1 and 2 orthologues define the specificity determinants that recognize the negatively charged C-terminal tail of $\alpha$-tubulin, cleave its terminal tyrosine residue, and mediate association with microtubules in cells. Our structural information further offers a unique basis to rationally design various types of small molecules that modulate the activity of vasohibins: they suggest that the integration of carboxylate group and tyrosine or phenylalanine ring mimics in a ligand should be a favorable starting point to attain potency and specificity. As such, vasohibin inhibitors could be used as tool compounds in basic research experiments aimed at perturbing the function of microtubules in different cell types. They may even find their way into medical applications as abnormal tubulin detyrosination levels are associated with human diseases including cancer, heart disorders, and possibly brain dysfunctions $\mathbf{s}^{2,3,12-15,40}$.

One key and rather unexpected finding is that SVBP activates vasohibins. SVBP has been previously shown to act as a chaperone that increases the solubility, abundance and stability of vasohibins in cells $s^{23,24,38}$. While SVBP is not a classical chaperone since we and others ${ }^{38}$ found that it forms a stable complex with vasohibins, our data do not rule out that SVBP indeed helps vasohibins to properly fold. However, they also clearly suggest that this small protein represents a regulatory element of vasohibin activity and that its interaction with vasohibins is required for normal axon development. In this context, different types of posttranslational modifications of vasohibins or SVBP would offer mechanisms to regulate the interaction between the two proteins and hence the activity of vasohibins in a spatiotemporal fashion to form detyrosinated microtubule subpopulations in cells.

One known posttranslational processing event of vasohibin is proteolytic cleavage within its $\mathrm{N}$ terminal domain resulting in truncated variants of vasohibin ${ }^{42}$. Based on our data, we expect that such deletions should impair SVBP binding and hence the stability and enzymatic activity of vasohibin. An attractive way to regulate the vasohibin-SVBP interaction would be phosphorylation. Interestingly, 
Y40 of SVBP, which is highly conserved across species, is annotated to be phosphorylated in the PhosphositePlus database ${ }^{43}$. The presence of a negatively-charged phosphate group on this tyrosine that is buried in the V2c-SVBP complex structure is predicted to severely affect the interaction of SVBP with vasohibins and therefore to modulate their activity. More work is needed to investigate this intriguing idea and to find the specific kinase and phosphatase system(s) involved.

The tubulin detyrosination-tyrosination cycle has been discovered almost half a century ago; however, its mechanism has remained enigmatic despite of its high physiological relevance. With the structure solution of the vasohibin-SVBP enzyme complexes, structural information of an eraser (TCP), the writer (TTL) and a reader (CAP-Gly) of the tubulin tyrosine signal is finally available and offers for the first time a basis to understand the cycle at the atomic level (Figure 7). Our study thus contributes an important puzzle piece that now allows describing a detailed molecular picture of an intriguing set of cytoskeletal enzymes and accessory proteins that regulate the physiology of cells and organs, and whose malfunctioning leads to disease. 


\section{Acknowledgments}

We thank N.E. Duggan, F. Vossier and L. Demacedo for technical assistance and the zootechnicians of the Grenoble Institute Neuroscience (GIN) for animal care. We thank Jacques Brocard for valuable discussions on data quantification. X-ray data were collected at beamlines BL-18U/19U of Shanghai Synchrotron Radiation Facility (Shanghai, China) and X06DA of the Swiss Light Source (Paul Scherrer Institut, Villigen, Switzerland). This work was supported by grants from the National Key R\&D Program of China (2018YFC1004500), PIC-GIN (Photonic Imaging Center of GIN, Univ Grenoble Alpes - Inserm U1216, which is part of the ISdV core facility and certified by the IBiSA label), INSERM, CEA, University Grenoble Alpes, CNRS, La Ligue Contre le Cancer comité de I'Isère (to MJM), fondation France Alzheimer (to MJM), the Swiss National Science Foundation (31003A_166608; to M.O.S.), the Shenzhen Government ('Peacock Plan' No. Y01226136; to H.H.), and the Thousand Young Talents Program (to H.H.).

\section{Author contributions}

N.W., designed and performed cloning experiments, prepared recombinant proteins, crystallized V2c-SVBP, V2c-SVBP-epoY, V2c-SVBP-TPCK, V2c-SVBP-maTail, V2-SVBP, and V1c-SVBP complexes, performed GST pull down experiments, analyzed the structures.

C.B., analyzed sequences, designed and performed cloning experiments for expression in mammalian cells.

S.R.C., analyzed sequences, designed and performed cloning experiments, prepared recombinant proteins, performed microtubule-binding experiments, crystallized V2c-SVBP complex, performed biophysical and biochemical experiments.

B.B., performed cycling cell-based experiments and statistical analyses.

L.P., performed neuronal experiments and statistical analyses.

N.O., analyzed sequences, designed and performed cloning experiments, prepared proteins.

H.B., collected and processed X-ray data on V2c-SVBP, V2c-SVBP-epoY, V2c-SVBP-TPCK, V2c-SVBPmaTail, V2-SVBP, and V1c-SVBP crystals.

V.O., collected and processed X-ray data, solved second V2c-SVBP structure.

F.K., performed in vitro detyrosination assays.

L.C., prepared some recombinant proteins.

A.A., provided intellectual input to the project. 
M.-J.M., performed enzymatic activity measurements, supervised the functional experiments with mammalian cells, analyzed all the data and wrote the paper with the input from all authors.

M.O.S., supervised crystallography and biochemistry experiments, analyzed all the data and wrote the paper with the input from all authors.

H.H., supervised crystallography and biochemistry experiments, analyzed all the data and wrote the paper with the input from all authors.

\section{Competing interest statement}

The authors declare no competing interests. 


\section{References}

1. Barisic, M., Silva e Sousa, Tripathy, S.K., Magiera, M.M., Zaytsev, A.V., Pereira, A.L., Janke, C., Grishchuk, E.L., \& Maiato, H., Mitosis. Microtubule detyrosination guides chromosomes during mitosis, Science 348, 799-803 (2015).

2. Robison, P., Caporizzo, M.A., Ahmadzadeh, H., Bogush, A.I., Chen, C.Y., Margulies, K.B., Shenoy, V.B., \& Prosser, B.L., Detyrosinated microtubules buckle and bear load in contracting cardiomyocytes, Science 352, aaf0659 (2016).

3. Chen, C.Y., Caporizzo, M.A., Bedi, K., Vite, A., Bogush, A.I., Robison, P., Heffler, J.G., Salomon, A.K., Kelly, N.A., Babu, A., Morley, M.P., Margulies, K.B., \& Prosser, B.L., Suppression of detyrosinated microtubules improves cardiomyocyte function in human heart failure, Nat Med. 24, 1225-1233 (2018).

4. Gobrecht, P., Andreadaki, A., Diekmann, H., Heskamp, A., Leibinger, M., \& Fischer, D., Promotion of Functional Nerve Regeneration by Inhibition of Microtubule Detyrosination, J Neurosci. 36, 3890-3902 (2016).

5. Marcos, S., Moreau, J., Backer, S., Job, D., Andrieux, A., \& Bloch-Gallego, E., Tubulin tyrosination is required for the proper organization and pathfinding of the growth cone, 4 , e5405 (2009).

6. Kerr, J.P., Robison, P., Shi, G., Bogush, A.I., Kempema, A.M., Hexum, J.K., Becerra, N., Harki, D.A., Martin, S.S., Raiteri, R., Prosser, B.L., \& Ward, C.W., Detyrosinated microtubules modulate mechanotransduction in heart and skeletal muscle, Nat Commun. 6, 8526 (2015).

7. Badin-Larcon, A.C., Boscheron, C., Soleilhac, J.M., Piel, M., Mann, C., Denarier, E., FourestLieuvin, A., Lafanechere, L., Bornens, M., \& Job, D., Suppression of nuclear oscillations in Saccharomyces cerevisiae expressing Glu tubulin, Proc. Natl. Acad. Sci. U. S. A 101, 5577-5582 (2004).

8. Konishi, Y. \& Setou, M., Tubulin tyrosination navigates the kinesin-1 motor domain to axons, Nat. Neurosci. 12, 559-567 (2009).

9. Peris, L., Thery, M., Faure, J., Saoudi, Y., Lafanechere, L., Chilton, J.K., Gordon-Weeks, P., Galjart, N., Bornens, M., Wordeman, L., Wehland, J., Andrieux, A., \& Job, D., Tubulin tyrosination is a major factor affecting the recruitment of CAP-Gly proteins at microtubule plus ends, J Cell Biol. 174, 839-849 (2006).

10. Tas, R.P., Chazeau, A., Cloin, B.M.C., Lambers, M.L.A., Hoogenraad, C.C., \& Kapitein, L.C., Differentiation between Oppositely Oriented Microtubules Controls Polarized Neuronal Transport, Neuron 96, 1264-1271 (2017).

11. Gundersen, G.G. \& Bulinski, J.C., Distribution of tyrosinated and nontyrosinated alpha-tubulin during mitosis, J Cell Biol. 102, 1118-1126 (1986).

12. Erck, C., Peris, L., Andrieux, A., Meissirel, C., Gruber, A.D., Vernet, M., Schweitzer, A., Saoudi, Y., Pointu, H., Bosc, C., Salin, P.A., Job, D., \& Wehland, J., A vital role of tubulin-tyrosine-ligase for neuronal organization, Proc. Natl. Acad. Sci. U. S. A 102, 7853-7858 (2005).

13. Lafanechere, L., Courtay-Cahen, C., Kawakami, T., Jacrot, M., Rudiger, M., Wehland, J., Job, D., \& Margolis, R.L., Suppression of tubulin tyrosine ligase during tumor growth, J. Cell Sci. 111 (Pt 2), 171-181 (1998). 
14. Whipple, R.A., Matrone, M.A., Cho, E.H., Balzer, E.M., Vitolo, M.I., Yoon, J.R., loffe, O.B., Tuttle, K.C., Yang, J., \& Martin, S.S., Epithelial-to-mesenchymal transition promotes tubulin detyrosination and microtentacles that enhance endothelial engagement, Cancer Res. 70, 8127-8137 (2010).

15. Belmadani, S., Pous, C., Ventura-Clapier, R., Fischmeister, R., \& Mery, P.F., Post-translational modifications of cardiac tubulin during chronic heart failure in the rat, Mol Cell Biochem. 237, 39-46 (2002).

16. Magiera, M.M., Singh, P., Gadadhar, S., \& Janke, C., Tubulin Posttranslational Modifications and Emerging Links to Human Disease, Cell 173, 1323-1327 (2018).

17. Westermann, S. \& Weber, K., Post-translational modifications regulate microtubule function, Nat. Rev. Mol. Cell Biol. 4, 938-947 (2003).

18. Nieuwenhuis, J. \& Brummelkamp, T.R., The Tubulin Detyrosination Cycle: Function and Enzymes, Trends Cell Biol. 29, 80-92 (2019).

19. Murofushi, H., Purification and characterization of tubulin-tyrosine ligase from porcine brain, J. Biochem. 87, 979-984 (1980).

20. Ersfeld, K., Wehland, J., Plessmann, U., Dodemont, H., Gerke, V., \& Weber, K., Characterization of the tubulin-tyrosine ligase, J. Cell Biol. 120, 725-732 (1993).

21. Prota, A.E., Magiera, M.M., Kuijpers, M., Bargsten, K., Frey, D., Wieser, M., Jaussi, R., Hoogenraad, C.C., Kammerer, R.A., Janke, C., \& Steinmetz, M.O., Structural basis of tubulin tyrosination by tubulin tyrosine ligase, J. Cell Biol. 200, 259-270 (2013).

22. Szyk, A., Deaconescu, A.M., Piszczek, G., \& Roll-Mecak, A., Tubulin tyrosine ligase structure reveals adaptation of an ancient fold to bind and modify tubulin, Nat. Struct. Mol. Biol. 18, 1250-1258 (2011).

23. Nieuwenhuis, J., Adamopoulos, A., Bleijerveld, O.B., Mazouzi, A., Stickel, E., Celie, P., Altelaar, M., Knipscheer, P., Perrakis, A., Blomen, V.A., \& Brummelkamp, T.R., Vasohibins encode tubulin detyrosinating activity, Science 358, 1453-1456 (2017).

24. Aillaud, C., Bosc, C., Peris, L., Bosson, A., Heemeryck, P., van, D.J., Le, F.J., Boulan, B., Vossier, F., Sanman, L.E., Syed, S., Amara, N., Coute, Y., Lafanechere, L., Denarier, E., Delphin, C., Pelletier, L., Humbert, S., Bogyo, M., Andrieux, A., Rogowski, K., \& Moutin, M.J., Vasohibins/SVBP are tubulin carboxypeptidases (TCPs) that regulate neuron differentiation, Science 358, 1448-1453 (2017).

25. Bieling, P., Kandels-Lewis, S., Telley, I.A., van, D.J., Janke, C., \& Surrey, T., CLIP-170 tracks growing microtubule ends by dynamically recognizing composite EB1/tubulin-binding sites, J Cell Biol. 183, 1223-1233 (2008).

26. Peris, L., Wagenbach, M., Lafanechere, L., Brocard, J., Moore, A.T., Kozielski, F., Job, D., Wordeman, L., \& Andrieux, A., Motor-dependent microtubule disassembly driven by tubulin tyrosination, J. Cell Biol. 185, 1159-1166 (2009).

27. Kreitzer, G., Liao, G., \& Gundersen, G.G., Detyrosination of tubulin regulates the interaction of intermediate filaments with microtubules in vivo via a kinesin-dependent mechanism, Mol. Biol. Cell 10, 1105-1118 (1999). 
28. Dunn, S., Morrison, E.E., Liverpool, T.B., Molina-Paris, C., Cross, R.A., Alonso, M.C., \& Peckham, M., Differential trafficking of Kif5c on tyrosinated and detyrosinated microtubules in live cells, J. Cell Sci. 121, 1085-1095 (2008).

29. Steinmetz, M.O. \& Akhmanova, A., Capturing protein tails by CAP-Gly domains, Trends Biochem. Sci. 33, 535-545 (2008).

30. Sanchez-Pulido, L. \& Ponting, C.P., Vasohibins: new transglutaminase-like cysteine proteases possessing a non-canonical Cys-His-Ser catalytic triad, Bioinformatics 32, 1441-1445 (2016).

31. Holm, L. \& Laakso, L.M., Dali server update, Nucleic Acids Res. 44, W351-W355 (2016).

32. Chatterjee, D., Boyd, C.D., O'Toole, G.A., \& Sondermann, H., Structural characterization of a conserved, calcium-dependent periplasmic protease from Legionella pneumophila, J Bacteriol. 194, 4415-4425 (2012).

33. Stieler, M., Weber, J., Hils, M., Kolb, P., Heine, A., Buchold, C., Pasternack, R., \& Klebe, G., Structure of active coagulation factor XIII triggered by calcium binding: basis for the design of next-generation anticoagulants, Angew. Chem Int. Ed Engl. 52, 11930-11934 (2013).

34. Xu, X., Li de la Sierra-Gallay, Kubiak, X., Duval, R., Chaffotte, A.F., Dupret, J.M., Haouz, A., \& Rodrigues-Lima, F., Insight into cofactor recognition in arylamine $\mathrm{N}$-acetyltransferase enzymes: structure of Mesorhizobium loti arylamine $\mathrm{N}$-acetyltransferase in complex with coenzyme A, Acta Crystallogr. D. Biol. Crystallogr. 71, 266-273 (2015).

35. D. S. Auld, "Carboxypeptidase A,"in Handbook of Proteolytic Enzymes (Third Edition), edited by N. D. Rawlings and G. alvesen (Academic Press, 2013), pp.1289-1301.

36. Watt, W., Koeplinger, K.A., Mildner, A.M., Heinrikson, R.L., Tomasselli, A.G., \& Watenpaugh, K.D., The atomic-resolution structure of human caspase-8, a key activator of apoptosis, Structure. 7, 1135-1143 (1999).

37. Kagawa, T.F., Cooney, J.C., Baker, H.M., McSweeney, S., Liu, M., Gubba, S., Musser, J.M., \& Baker, E.N., Crystal structure of the zymogen form of the group A Streptococcus virulence factor SpeB: an integrin-binding cysteine protease, Proc. Natl. Acad. Sci U. S. A 97, 2235-2240 (2000).

38. Suzuki, Y., Kobayashi, M., Miyashita, H., Ohta, H., Sonoda, H., \& Sato, Y., Isolation of a small vasohibin-binding protein (SVBP) and its role in vasohibin secretion, J Cell Sci 123, 3094-3101 (2010).

39. Saoudi, Y., Paintrand, I., Multigner, L., \& Job, D., Stabilization and bundling of subtilisintreated microtubules induced by microtubule associated proteins, J Cell Sci 108 ( Pt 1), 357367 (1995).

40. Sato, Y., The vasohibin family: a novel family for angiogenesis regulation, J Biochem. 153, 511 (2013).

41. Aillaud, C., Bosc, C., Saoudi, Y., Denarier, E., Peris, L., Sago, L., Taulet, N., Cieren, A., Tort, O., Magiera, M.M., Janke, C., Redeker, V., Andrieux, A., \& Moutin, M.J., Evidence for new Cterminally truncated variants of alpha- and beta-tubulins, Mol Biol. Cell 27, 640-653 (2016). 
42. Sonoda, H., Ohta, H., Watanabe, K., Yamashita, H., Kimura, H., \& Sato, Y., Multiple processing forms and their biological activities of a novel angiogenesis inhibitor vasohibin, Biochem. Biophys. Res. Commun. 342, 640-646 (2006).

43. Hornbeck, P.V., Zhang, B., Murray, B., Kornhauser, J.M., Latham, V., \& Skrzypek, E., PhosphoSitePlus, 2014: mutations, PTMs and recalibrations, Nucleic Acids Res. 43, D512D520 (2015). 


\section{Figure Legends}

\section{Fig. 1. Structural analysis of vasohibin-SVBP enzyme complex.}

a, Two overall views $60^{\circ}$ apart of the V2c-SVBP crystal structure in cartoon representation. The helical N-terminal domain (ND; cyan) of V2c wraps around SVBP (magenta). The C-terminal domain (CD; green) of V2c comprises the catalytic residues. The catalytic cysteine residue is colored in red and V2c secondary structure elements are labeled.

b-e, Side-by-side comparison of the V2c-SVBP structure in cartoon representation with structures of the representative transglutaminase-like cysteine protease superfamily members large adhesion protein G (LapG; PDB ID 4FGO), coagulation factor XIII (PDB ID 4KTY) and arylamine $\mathrm{N}$ acetyltransferase 1 (NAT1; PDB ID 4NV7). The equivalent catalytic core elements of the enzymes are highlighted in orange with red spheres highlighting their catalytic triads. Green spheres show the calcium ions that are essential for the activity of LapG (c) and coagulation factor XIII (d).

\section{Fig. 2. Vasohibin-SVBP active site architecture and molecular basis of inhibition.}

a, Surface view of V2c-SVBP color-coded with the electrostatic potential (red, negatively charged; blue, positively charged). The positively charged groove and the tyrosine-binding pocket are highlighted with red and green dashed circles, respectively.

b, Zoom in view on the active site of the V2c-SVBP structure in cartoon representation, with the catalytic residue triad shown in sticks representation.

c, Immunoblots of in cellulo tubulin detyrosination assays with selected active site V2 mutants and SVBP (see also Supplementary Fig. 2b). Plasmids encoding V2-eGFP and SVBP-myc-Flag were cotransfected into HEK293T cells. The "+" symbol refers to wild type protein. Non-transfected cells reveal the endogenous levels of detyrosinated tubulin. Uncropped images are shown in Supplementary Data Set 1.

d, Surface view of V2c-SVBP-epoY color-coded with the electrostatic potential. The inhibitor epoY is shown in sticks representation and is bound to the tyrosine-binding pocket.

e, Zoom in view on the active site of the V2c-SVBP-epoY structure. Interacting residues of V2c (green) with the ligand epoY (wheat) are shown in sticks representation. 
f, Immunoblots of in cellulo tubulin detyrosination assays with V1 and V2 mutants that were identified in the COSMIC database: V1 K145E, ID 1323382 (ovarian serous cystadenocarcinoma); V1 R222Q, ID 3498152 (caecum adenocarcinoma); V2 R211H, ID 425262 (colorectal carcinoma and breast invasive carcinoma, annotated as R167H). Uncropped images are shown in Supplementary Data Set 1.

g, Surface view of V2c-SVBP-TPCK color-coded with the electrostatic potential. TPCK is shown in sticks representation.

$h$, Zoom in view on the active site of the V2c-SVBP-TPCK structure. Interacting residues of V2c (green) with the ligand TPCK (wheat) are shown in sticks representation.

In (b), (e) and (h), hydrogen bonds are indicated by black dashed lines; water molecules are represented by yellow balls.

Fig. 3. Interaction of vasohibin-SVBP with a C-terminal $\alpha$-tubulin peptide.

a, Surface view of V2c-SVBP-maTail color-coded with the electrostatic potential (red, negatively charged; blue, positively charged). The segment 447-EGECY-451 of maTail was modeled in the structure and is highlighted in sticks representation. The catalytic residue C158 that forms a disulfide bond with maTail is labeled.

b, Zoom in view on the active site of the V2c-SVBP-maTail structure. Interacting residues of V2c (green) with maTail (wheat) are shown in sticks representation. Hydrogen bonds are indicated by dashed black lines.

c, Immunoblots of in cellulo tubulin detyrosination assays (presented as in Fig. 2c) with wild type V2 or V2 variants where residues interacting with maTail in the V2c-SVBP-maTail structure were mutated. Uncropped images are shown in Supplementary Data Set 1.

Fig. 4. Role of the vasohibin-SVBP interaction.

a-c, Zoom in views on the different contact points between V2c and SVBP (in cartoon representation) with interacting residues shown in sticks representation. In (c), V2c residues involved in shaping the 
active site are highlighted with red boxes. In (b) and (c), hydrogen bonds are indicated by black dashed lines; water molecules are represented by yellow balls.

d, SDS-PAGE of GST pull-down experiments performed with different MBP-V2c mutants and wild type GST-SVBP. The same set of mutants was also tested with full-length MBP-V2 (Supplementary Fig. $5 g)$.

e, SDS-PAGE of GST pull-down experiments performed with different GST-SVBP mutants and wild type MBP-V2c.

$\mathbf{f}, \mathbf{g}$, Immunoblots of in cellulo tubulin detyrosination assays (presented as in Fig. 2c) with wild type or mutated V2 and wild type SVBP (f), and with wild type or mutated SVBP and wild type V2 (g). Uncropped images for panels $(\mathbf{d}-\mathbf{g})$ are shown in Supplementary Data Set 1.

h, In vitro detyrosination activity assays performed with purified MBP-V2c or MBP-V2c $\triangle N D(0.2 \mu M$ each) in the absence or presence of purified SVBP $(0.4 \mu \mathrm{M})$ using $\left[{ }^{14} \mathrm{C}\right]$-tyrosinated taxol-stabilized microtubules $(2 \mu \mathrm{M})$. Data represent mean \pm SEM. $n=6,3,5$ and 6 independent experiments for $M B P-V 2 c+S V B P, M B P-V 2 c, M B P-V 2 c \triangle N D+S V B P$ and MBP-V2c $\triangle N D$, respectively.

i, In vitro detyrosination activity assays performed with purified MBP-V2c in the presence of purified wild type or mutated SVBP, and of purified SVBP in the presence of purified wild type or mutated MBP-V2c. $n=6,3,4$ and 4 for MBP-V2c + SVBP, MBP-V2c W63E W67E + SVBP, MBP-V2c L154E P155E + SVBP and V2c + SVBP I39A Y40A, respectively. Data represent mean \pm SEM. $n=6,4,4$ and 4 independent experiments for V2c + SVBP, V2c + SVBP I39A Y40A, V2c + SVBP W63E W67E and V2c + SVBP L154E P155E, respectively.

In (h) and (i) the theoretical 100\% tyrosine release level is indicated by a line. Source data for panels $\mathrm{h}$ and $\mathrm{i}$ are available in Supplementary Data Set 2.

Fig. 5. Determinants mediating association of the V2-SVBP complex with microtubules.

a, Surface representation of the V2c-SVBP structure, with V2c and SVBP shown in white and grey, respectively. Key residues of $\mathrm{V} 2 \mathrm{c}$ for tubulin detyrosination located in the active site and for a-tubulin tail contact are highlighted in purple. A cluster of four highly conserved basic residues on the surface of $\mathrm{V} 2 \mathrm{c}$ are highlighted in blue. 
b, Immunoblots of in cellulo tubulin detyrosination assays with wild type V2 or V2 4A (R137A K147A R151A H257A) and SVBP. Uncropped images are shown in Supplementary Data Set 1.

c, Representative immunofluorescence images of NIH3T3 cells cotransfected with plasmids encoding wild type V2-eGFP or V2 4A-eGFP and SVBP-myc-Flag. Merged images of V2-eGFP or V2 4A-eGFP and $\alpha$-tubulin stainings are displayed in the bottom lane. Insets are magnifications of the white square regions.

d, Quantification of the colocalisation between V2 proteins and microtubules. $n=12,29$ and 25 analyzed cells from three independent experiments for V2-eGFP, V2-eGFP + SVBP-Flag and V2-4AeGFP + SVBP-Flag, respectively. Data represent mean \pm SEM, ${ }^{* * * * P}<0.0001$, based on one-way ANOVA Dunn's Multiple Comparison Test.

$\mathbf{e}, \mathbf{f}, \mathbf{h}$, Microtubule pelleting assays. V2-SVBP wild type or mutants $(0.4 \mu \mathrm{M})$ were added to taxolstabilized microtubules at the tubulin concentrations indicated. The fit to the binding isotherm shown in (e) yielded a Kd of $0.16 \pm 0.04 \mu \mathrm{M}$. In (f) and (h), each dot represents an independent binding experiment of a V2-SVBP variant with intact (MT; $\mathbf{f}$ ) or subtilisin treated microtubules (sMT; $\mathbf{h}$ ) at concentrations of 0.2 and $2 \mu \mathrm{M}$. Data represent mean \pm SEM, $n=3$ independent experiments. ${ }^{* *} \mathrm{P}<0.002,{ }^{* * *} \mathrm{P}<0.0002$ based on one-way ANOVA Turkey's Multiple Comparison Test.

g, In vitro detyrosination activity assays performed with wild type V2 or V2 4A in the presence of wild type SVBP. Activity was assessed using $2 \mu \mathrm{M}\left[{ }^{14} \mathrm{C}\right]$-tyrosinated taxol-stabilized microtubules and a complex concentration of 50 or $100 \mathrm{nM}$. Data represent mean \pm SEM. $n=3$ independent experiments for both mutants and both concentrations.

Source data for panels (d-h) are available in Supplementary Data Set 2.

Fig. 6. Role of vasohibin-SVBP-microtubule interaction in the regulation of axon specification.

a, Neurons were transfected with plasmids allowing expression of shRNAs targeting mSVBP without or with plasmids encoding human hSVBP-myc-Flag or hSVBP I39A Y40A-myc-Flag and were analyzed by immunostaining at 1DIV (see Supplementary Fig. 7a). Stage III neurons (bearing an axon) were counted manually on immunofluorescence images. Proportions of stage III neurons (an index of neuronal differentiation) are represented (mean \pm SEM, $* * * * p<0.0001$ based on one Way ANOVA with Sidak's multiple comparison test $\overline{\overline{7}}_{\mathbf{7}} \cdot \mathrm{n}=6,7,7$ and 7 independent cell cultures for shRNA CTRL, 
shRNA mSVBP, shRNA mSVBP + hSVBP-myc-Flag and shRNA mSVBP + hSVBP I39A Y40A-myc-Flag, respectively.

b-c, Neurons were transfected with plasmids encoding wild type or mutated versions of V2, and analyzed by immunostaining at 1DIV. Percentage of stage III neurons (b) and of cells bearing two axons (c) were counted manually on immunofluorescence images and represented (mean \pm SEM, $* * * * p<0.0001$, based on one Way ANOVA with Sidak's multiple comparison test). $n=6,6,5$ and 5 independent cell cultures respectively for eGFP, V2-eGFP, V2 C158A-eGFP and V2 4A-eGFP. For each culture, more than 100 neurons were analyzed.

d, Representative images of stage III neurons bearing one axon (expressing eGFP, V2 C158A-eGFP or V2 4A-eGFP; highlighted by arrowheads) or two axons (expressing wild type V2-eGFP; highlighted by arrows). Neurons were transfected as in (b) and (c), tripled stained at 1DIV and analyzed by immunofluorescence for levels of V2 proteins (anti-eGFP antibody, top row) and for tyrosinated tubulin (red) and Tau content (axons, green) (bottom row).

Source data for (a-c) are available in Supplementary Data Set 2.

Fig. 7: Structural basis of the tubulin detyrosination-tyrosination cycle.

Most $\alpha$-tubulin isotypes of the $\alpha \beta$-tubulin heterodimer are synthesized in cells with a C-terminal glutamate-glutamate-tyrosine (EEY) motif (i). These EEY motifs are specifically recognized by proteins containing CAP-Gly domains like CLIP-170 or by kinesin motors like MCAK (ii). The terminal tyrosine of $\alpha$-tubulin can be removed by the vasohibin family of tubulin tyrosine carboxypeptidases (TCPs) (iii), and eventually added back by tubulin tyrosine ligase (TTL) (iv), which closes the cycle. As such, CAPGly and kinesin, TCP, and TTL can be considered as "reader", "eraser" and "writer" of the tubulin tyrosine signal that is eliminated or produced by the tubulin detyrosination-tyrosination cycle. Note that TTL is more active on free tubulin dimers while TCP is more active on microtubules ${ }^{23,24}$. 
Table 1: Data collection, phasing and refinement statistics for SAD structure

SeMet V2c-SVBP

(6J40)

\begin{tabular}{|c|c|}
\hline \multicolumn{2}{|l|}{ Data collection } \\
\hline Space group & $F 222$ \\
\hline \multicolumn{2}{|l|}{ Cell dimensions } \\
\hline$a, b, c(\AA)$ & $83.1,123.2,195.2$ \\
\hline \multirow[t]{2}{*}{$\alpha, \beta, \gamma\left({ }^{\circ}\right)$} & $90,90,90$ \\
\hline & Peak \\
\hline Wavelength (Å) & 0.9785 \\
\hline Resolution $(\AA)^{\mathrm{a}}$ & $50-2.20(2.24-2.20)^{a}$ \\
\hline$R_{\text {pim }}(\%)$ & $5.8(45.9)$ \\
\hline$I / \sigma(I)$ & $12.2(2.0)$ \\
\hline$C C_{1 / 2}$ & $0.691^{b}$ \\
\hline Completeness (\%) & 99.9 (99.9) \\
\hline Redundancy & $6.5(5.6)$ \\
\hline
\end{tabular}

\section{Refinement}

Resolution ( $\AA$ )

47.31-2.30

No. reflections

21,587

$R_{\text {work }} / R_{\text {free }}$

$18.4 / 22.4$

No. atoms

Protein $\quad 2,306$

Ligand

GOL $\quad 30$

$\begin{array}{ll}\mathrm{PO}_{4} & 15\end{array}$

Water 232

$B$ factors

$\begin{array}{ll}\text { Protein } & 36.2\end{array}$ 
Ligand

GOL

$\mathrm{PO}_{4}$

79.1

Water

40.8

R.m.s deviations

Bond lengths ( $\AA$ )

0.010

Bond angles $\left({ }^{\circ}\right)$

1.05

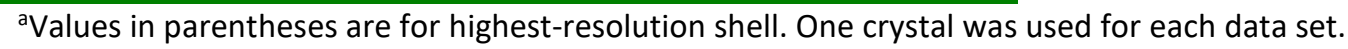

${ }^{b} C_{1 / 2}$ of the highest-resolution shell was shown. 
Table 2: Data collection and refinement statistics (molecular replacement)

\begin{tabular}{|c|c|c|c|}
\hline & $\begin{array}{l}\text { V2c-SVBP-epoY } \\
(6 \mathrm{~J} 4 \mathrm{P})\end{array}$ & $\begin{array}{l}\text { V2c-SVBP-TPCK } \\
\text { (6J4Q) }\end{array}$ & $\begin{array}{l}\text { V2c-SVBP-maTail } \\
(6 \mathrm{JV})\end{array}$ \\
\hline \multicolumn{4}{|l|}{ Data collection } \\
\hline Space group & $P 3221$ & $P 3221$ & $F 222$ \\
\hline \multicolumn{4}{|l|}{ Cell dimensions } \\
\hline$a, b, c(\AA)$ & $63.7,63.7,151.3$ & $117.2,117.2,209.0$ & $82.3,122.4,194.3$ \\
\hline$\alpha, \beta, \gamma\left(^{\circ}\right)$ & $90,90,120$ & $90,90,120$ & $90,90,90$ \\
\hline Resolution $(\AA)$ & $40-1.60(1.66-1.60)^{a}$ & $50-2.60(2.69-2.60)$ & $50-2.10(2.18-2.10)$ \\
\hline$R_{\text {pim }}(\%)$ & $2.6(7.5)$ & $3.0(58.5)$ & $4.0(19.6)$ \\
\hline$I / \sigma(I)$ & $26.8(11.1)$ & $25.6(1.5)$ & $12.8(1.7)$ \\
\hline$C C_{1 / 2}$ & $0.983^{b}$ & 0.621 & 0.866 \\
\hline Completeness (\%) & $100(100)$ & $100(100)$ & $99.0(91.2)$ \\
\hline Redundancy & $9.9(10.2)$ & $9.9(10.0)$ & $9.6(7.4)$ \\
\hline \multicolumn{4}{|l|}{ Refinement } \\
\hline Resolution $(\AA)$ & $37.22-1.60$ & $49.31-2.70$ & $38.05-2.10$ \\
\hline No. reflections & 47,942 & 43,495 & 28,518 \\
\hline$R_{\text {work }} / R_{\text {free }}$ & $16.4 / 18.8$ & $18.4 / 24.7$ & $16.9 / 20.0$ \\
\hline \multicolumn{4}{|l|}{ No. atoms } \\
\hline Protein & 2,390 & 8,966 & 2,348 \\
\hline \multicolumn{4}{|l|}{ Ligand } \\
\hline epoY & 23 & & \\
\hline TPCK & & 132 & \\
\hline GOL & & 30 & 24 \\
\hline $\mathrm{PO}_{4}$ & & & 10 \\
\hline Water & 478 & 251 & 239 \\
\hline \multicolumn{4}{|l|}{$B$ factors } \\
\hline Protein & 18.1 & 50.3 & 45.1 \\
\hline
\end{tabular}




\begin{tabular}{|c|c|c|c|}
\hline \multicolumn{4}{|l|}{ Ligand } \\
\hline epoY & 15.1 & & \\
\hline TPCK & & 64.1 & \\
\hline GOL & & 60.7 & 56.0 \\
\hline $\mathrm{PO}_{4}$ & & & 93.4 \\
\hline Water & 29.1 & 39.7 & 50.7 \\
\hline \multicolumn{4}{|l|}{ R.m.s. deviations } \\
\hline Bond lengths $(\AA ̊)$ & 0.006 & 0.010 & 0.007 \\
\hline Bond angles $\left({ }^{\circ}\right)$ & 0.97 & 1.19 & 0.79 \\
\hline
\end{tabular}

${ }^{a}$ Values in parentheses are for highest-resolution shell. One crystal was used for each data set.

${ }^{b} C C_{1 / 2}$ of the highest-resolution shell was shown. 
Table 3: Data collection and refinement statistics (molecular replacement)

\begin{tabular}{|c|c|c|c|}
\hline & $\begin{array}{l}\text { V2-SVBP } \\
\text { (6J4S) }\end{array}$ & $\begin{array}{l}\text { V1c-SVBP } \\
(6 \mathrm{JU})\end{array}$ & $\begin{array}{l}\text { V2c-SVBPc } \\
\text { (6QBY) }\end{array}$ \\
\hline \multicolumn{4}{|l|}{ Data collection } \\
\hline Space group & $C 222_{1}$ & $P 22_{1} 2_{1}$ & $P 3_{1} 21$ \\
\hline \multicolumn{4}{|l|}{ Cell dimensions } \\
\hline$a, b, c(\AA)$ & $74.2,111.5,127.2$ & $44.5,71.5,128.1$ & $119.9,119.9,102.6$ \\
\hline$\alpha, \beta, \gamma\left({ }^{\circ}\right)$ & $90,90,90$ & $90,90,90$ & $90,90,120$ \\
\hline Resolution ( $\AA$ ) & $50-2.70(2.80-2.70)^{a}$ & $50-2.00(2.07-2.00)$ & $104-2.11(2.34-2.11)$ \\
\hline$R_{\text {pim }}(\%)$ & $4.8(50.5)$ & $2.3(35.8)$ & $3.1(46.5)$ \\
\hline $1 / \sigma(I)$ & $15.8(1.5)$ & $24.6(1.2)$ & $19.2(1.7)$ \\
\hline$C C_{1 / 2}$ & $0.563^{b}$ & 0.738 & 0.681 \\
\hline Completeness (\%) & $99.7(97.4)$ & $99.9(99.5)$ & $94.5(78.5)^{d}$ \\
\hline Redundancy & $10.3(6.3)$ & $12.4(9.5)$ & $19.9(16.9)$ \\
\hline \multicolumn{4}{|l|}{ Refinement } \\
\hline Resolution $(\AA)$ & $44.30-2.80$ & $47.70-2.00$ & $104-2.11$ \\
\hline No. reflections & 12,747 & 28,433 & 30,812 \\
\hline$R_{\text {work }} / R_{\text {free }}$ & $17.2 / 22.7$ & $16.8 / 20.4$ & $19.0 / 23.0$ \\
\hline \multicolumn{4}{|l|}{ No. atoms } \\
\hline Protein & 2,309 & 2,251 & 4,442 \\
\hline \multicolumn{4}{|l|}{ Ligand } \\
\hline \multicolumn{4}{|l|}{ epoY } \\
\hline \multicolumn{4}{|l|}{ TPCK } \\
\hline GOL & 18 & & \\
\hline $\mathrm{PO}_{4}$ & 5 & & \\
\hline Water & 68 & 238 & 265 \\
\hline \multicolumn{4}{|l|}{$B$ factors } \\
\hline Protein & 42.8 & 56.9 & 53.5 \\
\hline
\end{tabular}




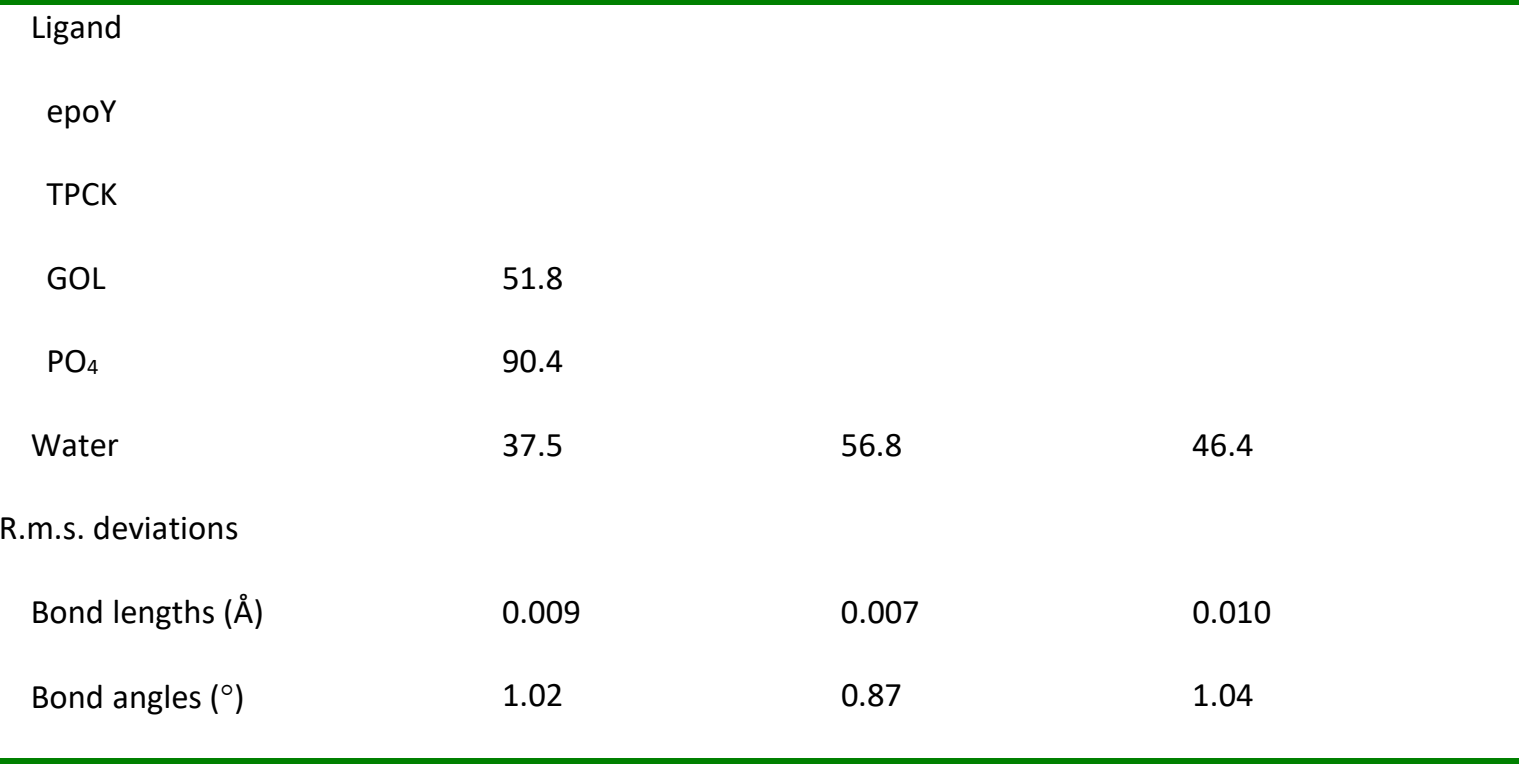

aValues in parentheses are for highest-resolution shell. One crystal was used for each data set.

${ }^{b} C C_{1 / 2}$ of the highest-resolution shell was shown.

'The second structure of V2c-SVBP obtained from one crystal in a different condition.

${ }^{d}$ For $6 Q B Y$ dataset only, this dataset was processed anisotropically with the ellipsoidal completeness. 


\section{Online Methods}

\section{Cloning and protein preparation in bacteria}

Full length human V2, V1 and SVBP cDNAs were isolated from a brain cDNA library. The cDNAs encoding full length V2 (residues 1-355), V2c (residues 46-296), and V1c (residues 57-306) were respectively cloned into the first multiple cloning site of a modified RSFDuet-1 vector (Novagen) with an N-terminal His6-SUMO tag. Full length human SVBP (residues 1-66) was cloned into the second multiple cloning site of the same vector for the co-expression of V2-SVBP, V2c-SVBP and V1c-SVBP complexes, respectively. All mutations were introduced using a standard PCR procedure and verified by DNA sequencing (GENEWIZ).

For expression of the various V2-SVBP, V2c-SVBP and V1c-SVBP complexes, BL21(DE3)-RIL (Stratagene) E. coli cells were first transformed with the corresponding RSFDuet-1 vectors and then cultured in Luria-Bertani (LB) medium with proper antibiotics at $37^{\circ} \mathrm{C}$ to an $\mathrm{OD}_{600}$ of 1.0-1.2. Protein expression was induced with $0.5 \mathrm{mM}$ IPTG and cells were further incubated overnight at $16{ }^{\circ} \mathrm{C}$. For expression of selenomethionine (SeMet)-labeled V2c-SVBP, cells were cultured in LeMaster and Richards minimal (LR) medium at $37^{\circ} \mathrm{C}$ to an $\mathrm{OD}_{600}$ of 1.0-1.2. Then the amino acids Val, lle, Leu, Phe, Thr, Trp and Lys at a final concentration of $50 \mathrm{mg} / \mathrm{L}$ and SeMet at a final concentration of $60 \mathrm{mg} / \mathrm{L}$ were added to the culture. After $30 \mathrm{~min}$ of incubation at $37^{\circ} \mathrm{C}$, cells were induced for expression with $0.5 \mathrm{mM}$ IPTG and further incubated overnight at $16^{\circ} \mathrm{C}$. The expressed protein complexes were first purified with $\mathrm{Ni}$-sepharose affinity beads (GE Healthcare). After removing the His 6 -SUMO tag by UIp1 (SUMO protease), the proteins were further purified on Heparin HP and HiLoad 16/600 Superdex 200 columns (GE Healthcare).

Codon optimized human V2 and SVBP genes were also synthesized (GENEWIZ). The codon optimized V2c (residues 40-295) cDNA was cloned into a pET3d vector (Novagen) without any fusion tag, while the codon optimized SVBP was cloned into a pET28 vector with a C-terminal $\mathrm{His}_{6}$ tag. The codon optimized V2c (residues 40-295; in pET3d) and SVBP (in pET28) were co-tranformed into BL21(DE3) E.coli cells to get a second V2c-SVBP complex. Transformed cells were then grown in LB media at $37{ }^{\circ} \mathrm{C}$ to an OD600 of 0.4-0.6 upon which the cultures were cooled to $20^{\circ} \mathrm{C}$ and induced with $0.5 \mathrm{mM}$ IPTG. Cells were harvested $18 \mathrm{~h}$ post-induction by centrifugation at $4000 \times \mathrm{g}$ for $15 \mathrm{~min}$ and the pellet resuspended in lysis buffer ( $500 \mathrm{mM} \mathrm{NaCl}, 50 \mathrm{mM}$ HEPES pH 8.0, 10\% glycerol, $10 \mathrm{mM}$ imidazole, 2 $\mathrm{mM} \beta$-mercaptomethanol, and Roche cOmplete protease inhibitor cocktail) at $50 \mathrm{ml}$ per liter culture. Following cell lysis by sonication, the extract was clarified by centrifugation at $20,000 \times \mathrm{g}$ for $30 \mathrm{~min}$ then passed through a $0.4 \mu \mathrm{m}$ filter prior to purification. Purification of the His-tagged protein was performed using HiTrap HP Ni ${ }^{2+}$ sepharose column (GE healthcare) as per manufacturer's instructions. 
Protein peak fractions were pooled and a final polishing step was performed using HiLoad 16/600 Superdex 75 size exclusion chromatography column in $20 \mathrm{mM}$ Tris-Cl, pH 7.5, $150 \mathrm{mM} \mathrm{NaCl}, 2 \mathrm{mM}$ DTT. Purified protein sample was flash frozen in liquid nitrogen and stored in $-80^{\circ} \mathrm{C}$ until further use.

For GST pull-downs, V2, V2 W63E W67E, V2 L154E P155E, V2c, V2c W63E W67E, V2c L154E P155E, and V2c $\triangle N D$ (residues 85-296) cDNAs were cloned into a modified RSFDuet-1 vector with an Nterminal $\mathrm{His}_{6}$-SUMO-MBP tag to get $\mathrm{N}$-terminal MBP fusion proteins. The MBP-tagged proteins were purified in the same way as described above. SVBP and its mutants Q35A R36A, I39A Y40A, L42A N43A, V45A M46A and E50A F54A were cloned into the pGEX-6p-1 vector (GE Healthcare) to obtain $\mathrm{N}$-terminal GST fusion proteins. The GST-tagged proteins were first purified using Glutathione Sepharose 4B beads (GE Healthcare) and further purified on a HiLoad 16/600 Superdex 200 size exclusion column. In some cases, the GST tags were removed with $3 \mathrm{C}$ protease prior to the size exclusion chromatography step.

The CDNA encoding the human TUBA-1A/B C-terminal $\alpha$-tubulin maTail mutant peptide (431DYEEVGVDSVEGEGEEEGECY-451, with E450 was mutated to cysteine) was cloned into the pGEX-6p-1 vector. The GST-tagged maTail was purified with Glutathione Sepharose 4B beads. After removing the GST tag with 3 C protease, the resulting maTail peptide was further purified onto a HiLoad 16/600 Superdex 75 column.

\section{Crystallization, $X$-ray data collection and structure determination}

All protein samples used for crystallization were in the same buffer $(20 \mathrm{mM}$ Tris- $\mathrm{HCl}, \mathrm{pH} 7.5,200 \mathrm{mM}$ $\mathrm{NaCl}$ and if not otherwise stated at the same protein concentration of $20 \mathrm{mg} / \mathrm{mL}$. Crystals were obtained as follows:

(1) The SeMet-labeled V2c-SVBP was crystallized in $1.6 \mathrm{M}$ sodium-potassium phosphate, pH 8.2.

(2) To form the co-complexes of V2c-SVBP with epoY and TPCK, V2c-SVBP was mixed with the respective inhibitor at a mole ratio of 1:5 and incubated on ice for $30 \mathrm{~min}$. Then, the resulting cocomplexes V2c-SVBP-epoY and V2c-SVBP-TPCK were crystallized in $1.0 \mathrm{M}$ lithium chloride, $0.1 \mathrm{M}$ HEPES, pH 7.0, 20\% PEG 6000 or in $1.0 \mathrm{M}$ sodium malonate, pH 7.0, respectively.

(3) The V2-SVBP complex at a concentration of $15 \mathrm{mg} / \mathrm{mL}$ was crystallized in $1.8 \mathrm{M}$ sodium-potassium phosphate, $\mathrm{pH} 6.8$. 
(4) The wild type V1c-SVBP complex did not yield well diffracting crystals. We thus analyzed the crystal packing of V2c-SVBP and systematically mutated some residues of V1c to the corresponding ones of V2c, which are involved in crystal contacts. Notably, by introducing the three mutations E71S A72H K79M into V1c allowed us to solve the V1c-SVBP structure to high resolution. Well diffracting crystals of the mutant V1c-SVBP complex were obtained in $1.0 \mathrm{M}$ lithium chloride, $0.1 \mathrm{M}$ citric acid, pH 5.0, 20\% PEG 6000.

(5) For crystallization of V2c-SVBP C58S in complex with the maTail peptide the components were mixed in a 1:3 molar ratio and incubated at room temperature for $24 \mathrm{~h}$ before setting up crystallization trials. The resulting V2c-SVBP S58C-maTail complex was crystallized in $1.4 \mathrm{M}$ sodiumpotassium phosphate, $\mathrm{pH} 8.2$.

(6) A second V2c-SVBP complex using a slightly different V2c construct encoding for residues 40-295 of V2c was crystallized in $0.2 \mathrm{M}$ sodium formate, $0.1 \mathrm{M}$ bis-tris propane, $\mathrm{pH}$ 6.5, and 20\% PEG 3350.

All crystals were obtained using the sitting-drop vapor-diffusion method at $20{ }^{\circ} \mathrm{C}$ and soaked in cryoprotectants made from the mother liquors supplemented with $15-30 \%$ glycerol before flash freezing in liquid nitrogen.

The X-ray diffraction data for solving the structures of the SeMet V2c-SVBP, V2c-SVBP-maTail and V2-SVBP complexes were collected at $0.9785 \AA$ on beamline BL-18U, and the data for solving the structures of the V2c-SVBP-epoY, V2c-SVBP-TPCK and V1c-SVBP complexes were collected at 0.9789 $\AA$ at beamline BL-19U at the Shanghai Synchrotron Radiation Facility (SSRF), China. The native and ISAD data obtained with Nal soaked crystals for the structure of the second V2c-SVBP complex were collected at 1.0000 and $2.0750 \AA$, respectively, at beamline X06DA at the Swiss Light Source (SLS), Paul Scherrer Institut, Switzerland.

X-ray data sets were processed using HKL $3000^{46}$ or XDS and XSCALE ${ }^{47}$. The phase problem was solved by single-wavelength anomalous dispersion (SAD) method using SHELXC/D ${ }^{44}$ and PHENIX ${ }^{48}$ with the data obtained from one crystal of SeMet-labeled V2c-SVBP (12 selenium sites were located by SHELXC/D; Figure of merit (FOM) calculated from AutoSol in PHENIX was 0.358); for the second V2cSVBP complex structure, the phase problem was also solved by SAD with the X-ray data obtained from a Nal soaked crystal. In the latter case, the substructure consisting of 8 I atoms (SHELXD ${ }^{44}$ $\mathrm{CC}_{\text {all }} / \mathrm{CC}_{\text {weak }}$ 0.273/0.113) allowed successful phasing and automatic building of 542 residues (2 molecules in the asymmetric unit) using CRANK ${ }^{45}$. The initial respective models were manually corrected and refined in $\operatorname{Coot}^{49}$ and BUCCANEER ${ }^{50}$ and then refined using PHENIX and BUSTER (Global Phasing). After further iterative rounds of refinement, the final two structures of V2c-SVBP (favored 
$95.7 \%$, allowed $4.3 \%$ and favored $97.8 \%$, allowed $1.9 \%$, respectively) were refined to 2.30 and $2.09 \AA$, respectively.

The structures of V2c-SVBP-epoY, V2c-SVBP-TPCK, V2c-SVBP-maTail, V2-SVBP and V1c-SVBP were determined by molecular replacement in PHASER $^{51}$ with the structure of V2c-SVBP as the search model, and then were manually modified using Coot and refined in PHENIX. The final structures of V2c-SVBP-epoY (favored 97.9\%, allowed 2.1\%), V2c-SVBP-TPCK (favored 95.6\%, allowed 4.0\%), V2cSVBP-maTail (favored 96.4\%, allowed 3.2\%), V2-SVBP (favored 97.1\%, allowed 2.5\%), and V1c-SVBP (favored 95.6\%, allowed 4.4\%) were refined to 1.60, 2.70, 2.10, 2.80, and $2.00 \AA$, respectively. Data collection and refinement statistics are given in Table 1-3. Figures were prepared using PyMOL (The PyMOL Molecular Graphics System, Schrödinger).

\section{Isothermal titration calorimetry (ITC)}

Protein samples were buffer-exchanged to $20 \mathrm{mM}$ HEPES, pH 7.5, $150 \mathrm{mM} \mathrm{NaCl}$ and then supplemented with $0.5 \mathrm{mM}$ tris(2-carboxyethyl)phosphine (TCEP). ITC experiments were performed at $25{ }^{\circ} \mathrm{C}$ on an iTC200 calorimeter (MicroCal) using 15 injections of $2.6 \mu \mathrm{l}$. A $15 \mu \mathrm{M}$ solution of $\mathrm{N}$ terminal His 6 -SUMO-MBP tagged V2c was loaded in the cell. A $150 \mu \mathrm{M}$ solution of C-terminal His 6 tagged SVBP was loaded into the syringe. The measured heat changes of the binding reactions were integrated and processed using the standard "one set of sites" model implemented in the Origin software package (OriginLab) to determine the binding stoichiometry, $\mathrm{N}$, and the equilibrium dissociation constant, $\mathrm{Kd}$.

\section{Microtubule pelleting assay}

Microtubule pelleting assays were performed using lyophilized bovine brain tubulin purchased from the Centro de Investigaciones Biológicas, CSIC, Madrid, Spain. To generate taxol-stabilized microtubules, tubulin was solubilized at $2 \mathrm{mg} / \mathrm{ml}$ in BRB80 buffer (80 mM PIPES-KOH, pH 6.8, supplemented with $1 \mathrm{mM} \mathrm{MgCl}$ and $1 \mathrm{mM} \mathrm{EGTA}$,) containing $0.5 \mathrm{mM}$ GTP and $1.25 \mathrm{mM}$ DTT. The samples were incubated for $5 \mathrm{~min}$ on ice then for $10 \mathrm{~min}$ at $37^{\circ} \mathrm{C}$. Polymerization was induced by the successive addition of taxol (Sigma-Aldrich) to the reaction at $0.1 \mu \mathrm{M}, 1 \mu \mathrm{M}, 10 \mu \mathrm{M}$ final concentration for each step. 
To remove the C-terminal tails of tubulin, subtilisin (Sigma-Aldrich) was added to microtubules at a 1:1 ratio $(\mathrm{w} / \mathrm{w})$ and incubated for $1 \mathrm{hr}$ at $30{ }^{\circ} \mathrm{C}^{39}$. The reaction was terminated by the addition of PMSF to $2 \mathrm{mM}$ final concentration. The cleaved microtubules were sedimented through a taxolglycerol cushion $\left(20 \mu \mathrm{M}\right.$ taxol, $40 \%$ glycerol in BRB80) for $30 \mathrm{~min}$ at $30{ }^{\circ} \mathrm{C}$ and $300,000 \times \mathrm{g}$. Microtubule pellets were resuspended in BRB80 supplemented with $10 \mu \mathrm{M}$ taxol at $2 \mathrm{mg} / \mathrm{ml}$ concentration.

Taxol-stabilized microtubules (intact or subtilisin digested) and V2 protein variants were incubated together in BRB80 for $10 \mathrm{~min}$ at $25^{\circ} \mathrm{C}$. For all reactions, the final concentration of V2 variants was set constant at $0.4 \mu \mathrm{M}$ while the microtubule concentration was varied as indicated in the plots. The reaction mixtures were centrifuged through a taxol-glycerol cushion as described above to acquire the microtubule-enriched pellet fraction, which was then analyzed by $15 \%$ SDS-PAGE. Following Coomassie staining, gel densitometry analyses were performed using ImageJ (https://imagej.nih.gov/ij/index.html).

The band intensity value of V2 variants were normalized against control V2 bands of known concentration to obtain the percentage bound value. In the plot of V2-SVBP binding at various microtubule concentrations, the following equation was used for the fitting:

$$
\Delta \delta=\Delta \delta_{\max }\left(K_{d}+[P]_{t}+[L]_{t}-\left\{\left(K_{d}+[P]_{t}+[L]_{t}\right)^{2}-\left(4[P]_{t}[L]_{t}\right)\right\}^{1 / 2}\right) / 2[P]_{t}
$$

where $[\mathrm{P}]_{\mathrm{t}}$ is the concentration of $\mathrm{V} 2-\mathrm{SVBP},[\mathrm{L}]_{\mathrm{t}}$ is the concentration of tubulin heterodimers, and $\mathrm{Kd}$ is the dissociation constant.

\section{GST pull-down assays}

For the pull-downs of wild type GST-SVBP and derived mutants Q35A R36A, I39A Y40A, L42A N43A, V45A M46A and E50A F54A with MBP-V2c, $40 \mu \mathrm{L}$ of Glutathione Sepharose 4B beads were suspended with $200 \mu \mathrm{L}$ of binding buffer ( $20 \mathrm{mM}$ Tris- $\mathrm{HCl}$, pH 7.5, supplemented with $0.5 \mathrm{M} \mathrm{NaCl}$ ); and $1.5 \mathrm{nmol}$ of GST-SVBP protein variants were added. Then $1.5 \mathrm{nmol}$ of MBP-V2c were added and incubated at $4{ }^{\circ} \mathrm{C}$ for $2 \mathrm{~h}$. The beads were subsequently washed five times with $1 \mathrm{~mL}$ of washing buffer ( $20 \mathrm{mM}$ Tris- $\mathrm{HCl}, \mathrm{pH} 7.5$, supplemented with $0.5 \mathrm{M} \mathrm{NaCl}$ and $0.5 \%$ Triton X-100) before adding $50 \mu \mathrm{L}$ of sample buffer. An aliquot of $10 \mu \mathrm{L}$ of each sample was analyzed by $12 \%$ reducing SDS-PAGE. The GST pull-downs of GST-SVBP with MBP-tagged V2, V2 W63E W67E, V2 L154E P155E, V2c, V2c W63E W67E, V2C L154E P155E, and V2c $\triangle N D$ were performed in the same way. 


\section{In vitro tubulin detyrosination assays}

Enzyme activity was measured using $\left[{ }^{14} \mathrm{C}\right]$-tyrosinated taxol-stabilized microtubules $(2 \mu \mathrm{M}$ radiolabeled tubulin, prepared using recombinant $T T L$ from chicken ${ }^{21}$ ), and wild type or mutant V2 with or without wild type or mutant SVBP at the concentrations indicated in the legend to figures in $80 \mathrm{mM}$ Pipes- $\mathrm{KOH}, \mathrm{pH}$ 6.7, supplemented with $1 \mathrm{mM}$ EGTA and $1 \mathrm{mM} \mathrm{MgCl}$. Reactions were performed for the time indicated at $37{ }^{\circ} \mathrm{C}$, and were stopped on ice by addition of $20 \mu \mathrm{g} / \mathrm{ml}$ bovine serum albumin and $10 \%$ perchloric acid followed by centrifugation. The release of tyrosine from microtubules was estimated by measuring the radioactivity in the supernatant (a zero time control was subtracted).

Plasmids constructions for mammalian cells and antibodies for immunoblotting (IB) and immunofluorescence (IF)

Point mutations and deletions were introduced in human V1, V2 or SVBP CDNAs by standard PCR procedures. V1 and V2 amplicons were cloned into a home-made CAG promoter-containing vector generating proteins with eGFP at their C-terminus, using the In-Fusion HD cloning kit (ThermoFischer Scientific). SVBP amplicons were cloned into pCMV6-Entry vector generating proteins with myc and Flag tags at their C-terminus (PCR-encoded). All mutations were verified by DNA sequencing (Eurofins).

Total tubulin was detected using the $\alpha 3 \mathrm{~A} 1$ antibody (mouse, 1:10,000 in IF) and detyrosination activity was assayed using antibodies specific for tyrosinated (Tyr-tub or YL1/2, rat, 1:6,000 in IF) and detyrosinated $\alpha$-tubulin (deTyr-tub, rabbit , 1:5,000 in IB and 1:600 in IF, and deTyr-tub, guinea pig, $1: 600$ in IF), as described in ${ }^{24}$. Other primary antibodies were anti-Flag (rabbit 1:2,000 in IB and IF) from Molecular Probes, anti-GFP (rabbit, 1:5,000 in IB and 1:1,000 in IF) from Life Technologies, antiGFP (chicken, 1:2,000 in IF) from Millipore, anti GAPDH (rabbit, 1:10,000 in IB) from Sigma, and antiTau (mouse, 1:1,000 in IF) from Millipore.

Immunoblot images were acquired with a ChemiDoc MP Imaging System (Biorad). The quantification of immunoblots was performed using the Fiji software ${ }^{52}$. 


\section{Cycling cell experiments}

HEK293T were obtained from ATCC (ATCC CRL-3216) and NIH3T3 are lab-derived cells ${ }^{53}$. Cells were cultured at $37^{\circ} \mathrm{C}$ under $5 \% \mathrm{CO}_{2}$ in DMEM (ThermoFischer Scientific) containing $10 \%$ FCS and $1 \%$ penicillin and $1 \%$ streptomycin. Cells were regularly tested for mycoplasma contamination. HEK293Tcells were transfected with JetPRIME transfection reagent (Polyplus-Transfection). NIH3T3 cells were transfected with Lipofectamine transfection reagent (Invitrogen). A ratio of 1:1 was used for cDNA co-transfections (for V1 or V2 with SVBP).

For in cellulo tubulin detyrosination assay, HEK293T cells were collected $24 \mathrm{~h}$ after transfection. After washing with phosphate-buffered saline (PBS) medium at $37^{\circ} \mathrm{C}$, cells were either lysed in Laemmli buffer (crude extract) or lysed in $100 \mathrm{mM}$ Tris-HCl, pH 7.1, and 1\% Tx100 in the presence of a protease inhibitor cocktail (cOmplete EDTA-free, Roche) before being centrifuged for 20 min at $4{ }^{\circ} \mathrm{C}$ and 70,000 $\mathrm{g}$. Supernatants were then diluted in Laemmli buffer (soluble fraction). Protein extracts were loaded on 4-15\% gradient acrylamide gels (Mini-PROTEAN ${ }^{\circledR}$ TGX Stain-Free ${ }^{\text {TM }}$, Invitrogen) and transferred with Trans-Blot ${ }^{\circledast}$ Turbo (BioRad). Membranes were incubated with primary and secondary antibodies conjugated with HRP and finally revealed with Chemidoc ${ }^{\mathrm{TM}}$ MP Imaging Sytem (Biorad).

For in cellulo colocalization study, NIH3T3 cells were permeabilized $24 \mathrm{~h}$ after transfection by coverslips incubation in OPT medium (80 mM Pipes-KOH, pH 7.1, supplemented with $1 \mathrm{mM}$ EGTA, 1 $\mathrm{mM} \mathrm{MgCl} 2,0.5 \% \mathrm{Tx} 100$, and $10 \%$ glycerol) for $1 \mathrm{~min}$ at $37^{\circ} \mathrm{C}$ prior to fixation in methanol (10 $\mathrm{min}$ at $20^{\circ} \mathrm{C}$ ). Cells were then incubated with primary antibodies, followed by incubation with secondary antibodies conjugated with either alexa-488, cyanine-3 or cyanine-5 fluorophores $(1: 1,000)$. Nuclei were stained using Hoechst $33258(1 \mu \mathrm{g} / \mathrm{ml})$ directly diluted in mounting medium (Dako). Acquisitions of images were performed using the confocal microscope LSM770 (Zeiss) equipped with a 63x 1.4 NA objective. Quantification of the colocalization of V2 with $\alpha$-tubulin was carried out using the ImageJ plugin Jacop ${ }^{54}$.

All SDS-PAGE, immuno-blots or immunofluorescence data are representative results from independent experiments performed at least 3-times. HEK293T cells were transfected with JetPRIME transfection reagent (Polyplus-Transfection). NIH3T3 cells were transfected with Lipofectamine transfection reagent (Invitrogen). A ratio of 1:1 was used for cDNA co-transfections (for V1 or V2 with SVBP). 


\section{Neuron experiments}

Hippocampal neurons were prepared from SWISS mice embryos of 17,5 days (from 2-4 months mother mice, Janvier Labs) as described $\mathrm{in}^{24}$. The protocol was approved by the local animal welfare committee (Comité Local Grenoble Institute Neurosciences, C2EA-04) and complied to EU guidelines (directive 2010/63/EU). Every precaution was taken to minimize stress and the number of animals used for each experiment.

Hippocampal neurons were prepared and transfected using Amaxa Nucleofector kit (Lonza) as previously described $\mathrm{in}^{24}$. For each transfection point, 2 embryos were dissected. A ratio of 1:1 was used for cDNA co-transfections. shRNA experiments targeting mouse SVBP are described in ${ }^{24}$.

For IF, cells were fixed at $37{ }^{\circ} \mathrm{C}$ in $4 \%$ paraformaldehyde, $4.2 \%$ sucrose, phosphate buffered saline medium (PBS) and permeabilized using $0.1 \%$ Triton X-100, PBS. Cells were then incubated with primary antibodies, followed by incubation with secondary antibodies conjugated with either alexa488, cyanine-3 or cyanine-5 fluorophores $(1: 1,000)$. Nuclei were stained using Hoescht 33258 (1 $\mu \mathrm{g} / \mathrm{ml})$.

For morphometric neuron analysis, mosaic images of neurons cultured for 1 day in vitro (1DIV) and fixed prior to staining with anti-Tau and anti-Tyr tubulin antibodies were acquired with a 20x N.A 0.5 objective on a DMI6000 Leica microscope with a motorized stand. Neurons bearing zero, one or more than one Tau positive axon were manually counted. For each transfection point, more than 95 neurons were quantified in at least 2 different slices. The percentage of stage III cells were calculated as the sum of the percentages of neurons bearing one or more than one Tau positive axon.

\section{Data availability statement}

Coordinates of the X-ray crystal structures have been deposited in the RCSB PDB (www.rcsb.org) with the following accession numbers: V2c-SVBP (6J4O and 6QBY), V2c-SVBP-epoY (6J4P), V2c-SVBP-TPCK (6J4Q), V2c-SVBP-maTail (6J4V), V2-SVBP (6J4S), and V1c-SVBP (6J4U). Uncropped blot/gel images are shown in Supplementary Data Set 1 . Source data for Figs $4 \mathrm{~h}$, i, 5d-h, 6a-c, Suppl. Fig. 2, Suppl. Fig 4 and Suppl. Fig. $5 f$ are available in Supplementary Data Set 2. Other data are available upon reasonable request. 


\section{Online Methods-only references}

44. Sheldrick, G.M., Experimental phasing with SHELXC/D/E: combining chain tracing with density modification, Acta Crystallogr. D. Biol. Crystallogr. 66, 479-485 (2010).

45. Skubak, P. \& Pannu, N.S., Automatic protein structure solution from weak X-ray data, Nat Commun. 4, 2777 (2013).

46. Minor, W., Cymborowski, M., Otwinowski, Z., \& Chruszcz, M., HKL-3000: the integration of data reduction and structure solution--from diffraction images to an initial model in minutes, Acta Crystallogr. D. Biol. Crystallogr. 62, 859-866 (2006).

47. Kabsch, W., XDS, Acta Crystallogr. D. Biol. Crystallogr. 66, 125-132 (2010).

48. Adams, P.D., Afonine, P.V., Bunkoczi, G., Chen, V.B., Davis, I.W., Echols, N., Headd, J.J., Hung, L.W., Kapral, G.J., Grosse-Kunstleve, R.W., McCoy, A.J., Moriarty, N.W., Oeffner, R., Read, R.J., Richardson, D.C., Richardson, J.S., Terwilliger, T.C., \& Zwart, P.H., PHENIX: a comprehensive Python-based system for macromolecular structure solution, Acta Crystallogr. D. Biol. Crystallogr. 66, 213-221 (2010).

49. Emsley, P. \& Cowtan, K., Coot: model-building tools for molecular graphics, Acta Crystallogr. D. Biol. Crystallogr. 60, 2126-2132 (2004).

50. Cowtan, K., Completion of autobuilt protein models using a database of protein fragments, Acta Crystallogr. D. Biol. Crystallogr. 68, 328-335 (2012).

51. McCoy, A.J., Grosse-Kunstleve, R.W., Adams, P.D., Winn, M.D., Storoni, L.C., \& Read, R.J., Phaser crystallographic software, J Appl Crystallogr. 40, 658-674 (2007).

52. Schindelin, J., Arganda-Carreras, I., Frise, E., Kaynig, V., Longair, M., Pietzsch, T., Preibisch, S., Rueden, C., Saalfeld, S., Schmid, B., Tinevez, J.Y., White, D.J., Hartenstein, V., Eliceiri, K., Tomancak, P., \& Cardona, A., Fiji: an open-source platform for biological-image analysis, Nat. Methods 9, 676-682 (2012).

53. Lieuvin, A., Labbé, J.C., Dorée, M., Job, D., Intrinsic microtubule stability in interphase cells, J Cell Biol. 124, 985-996 (1994).

54. Bolte, S. \& Cordelieres, F.P., A guided tour into subcellular colocalization analysis in light microscopy, J Microsc. 224, 213-232 (2006). 


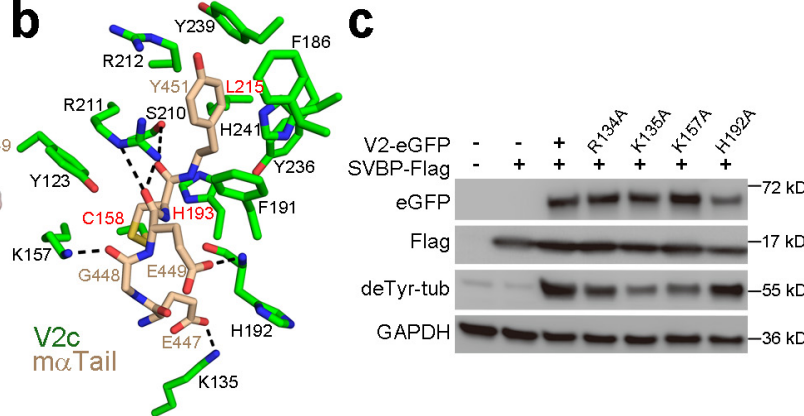



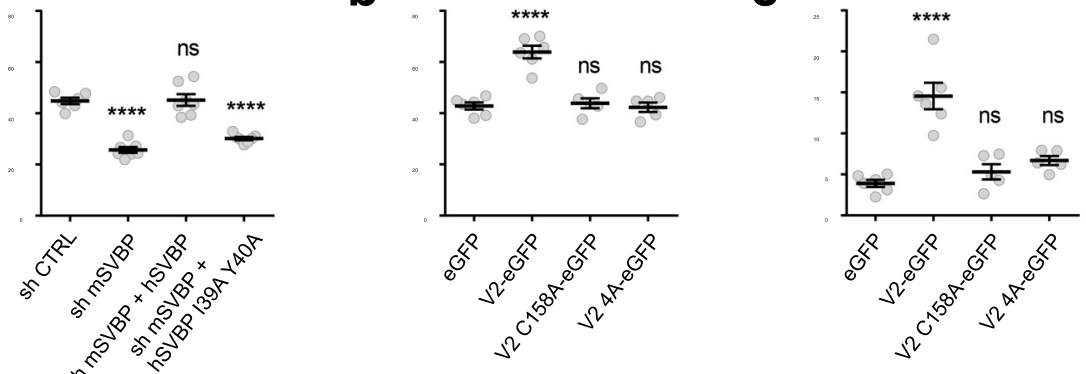

d

के

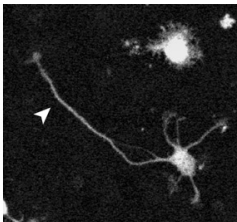

Niv
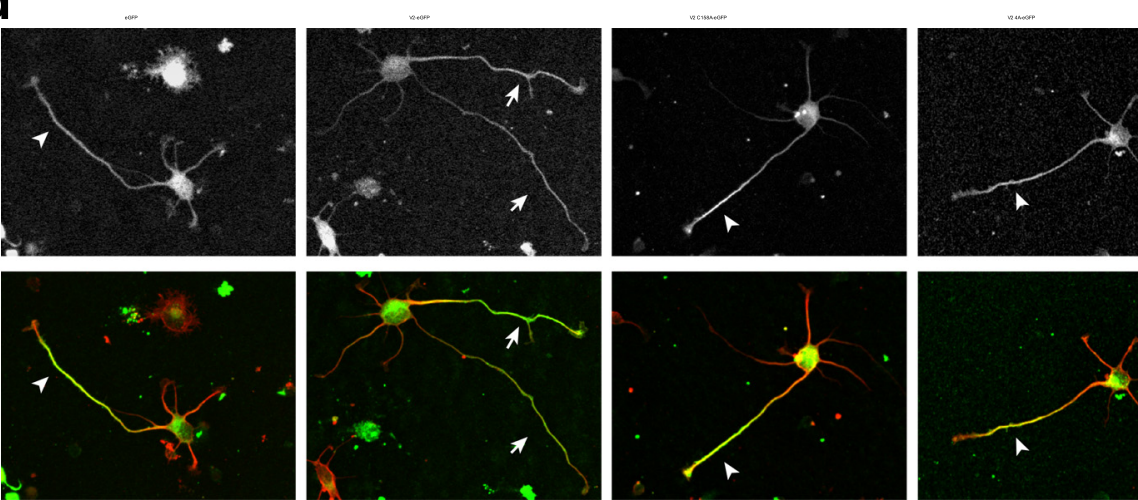

$20 \mu \mathrm{m}$ 


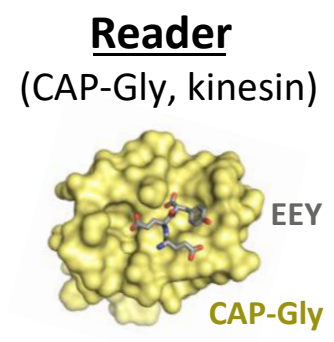

(ii)

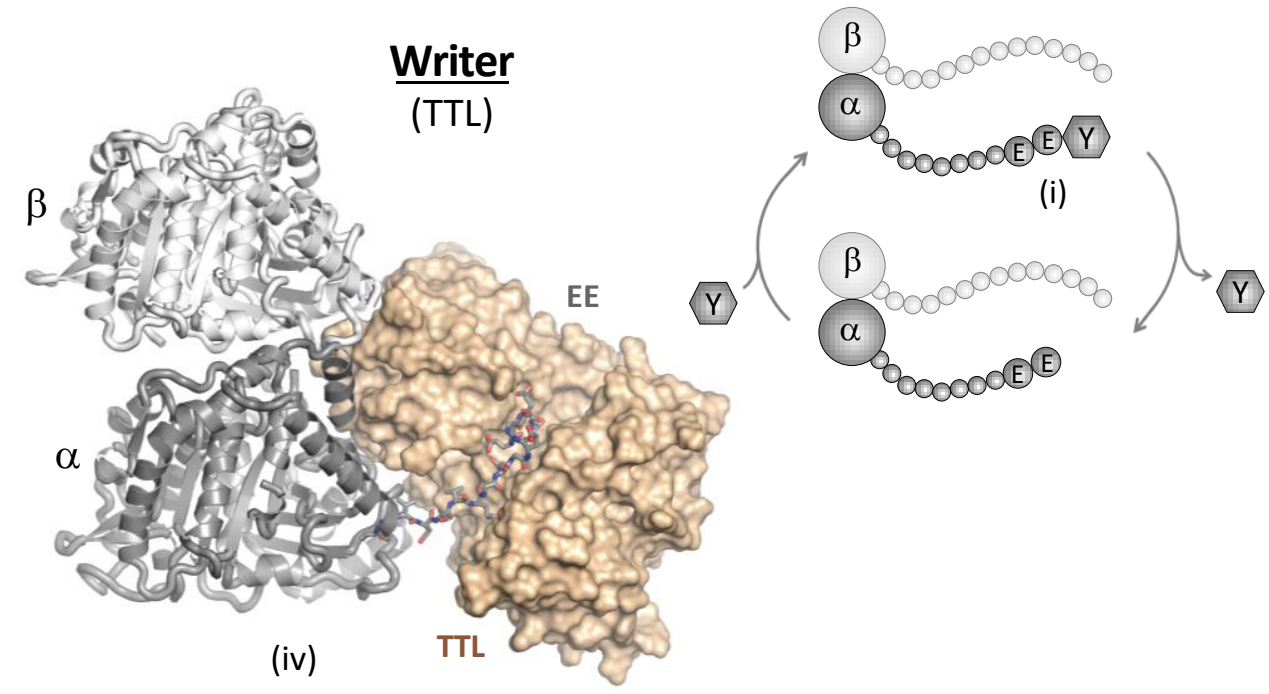

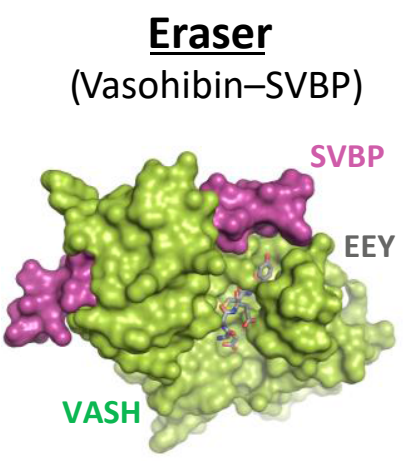

(iii) 


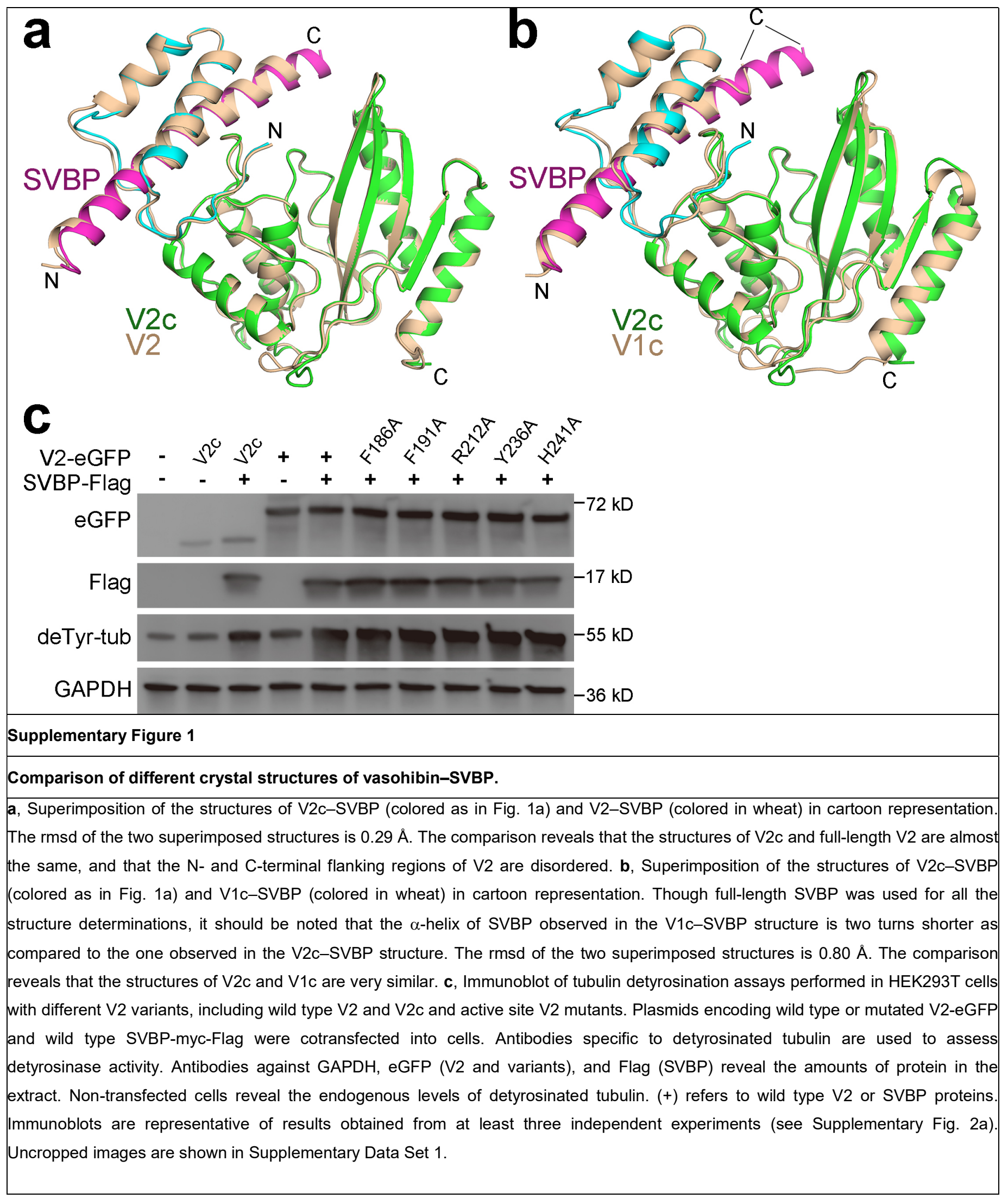




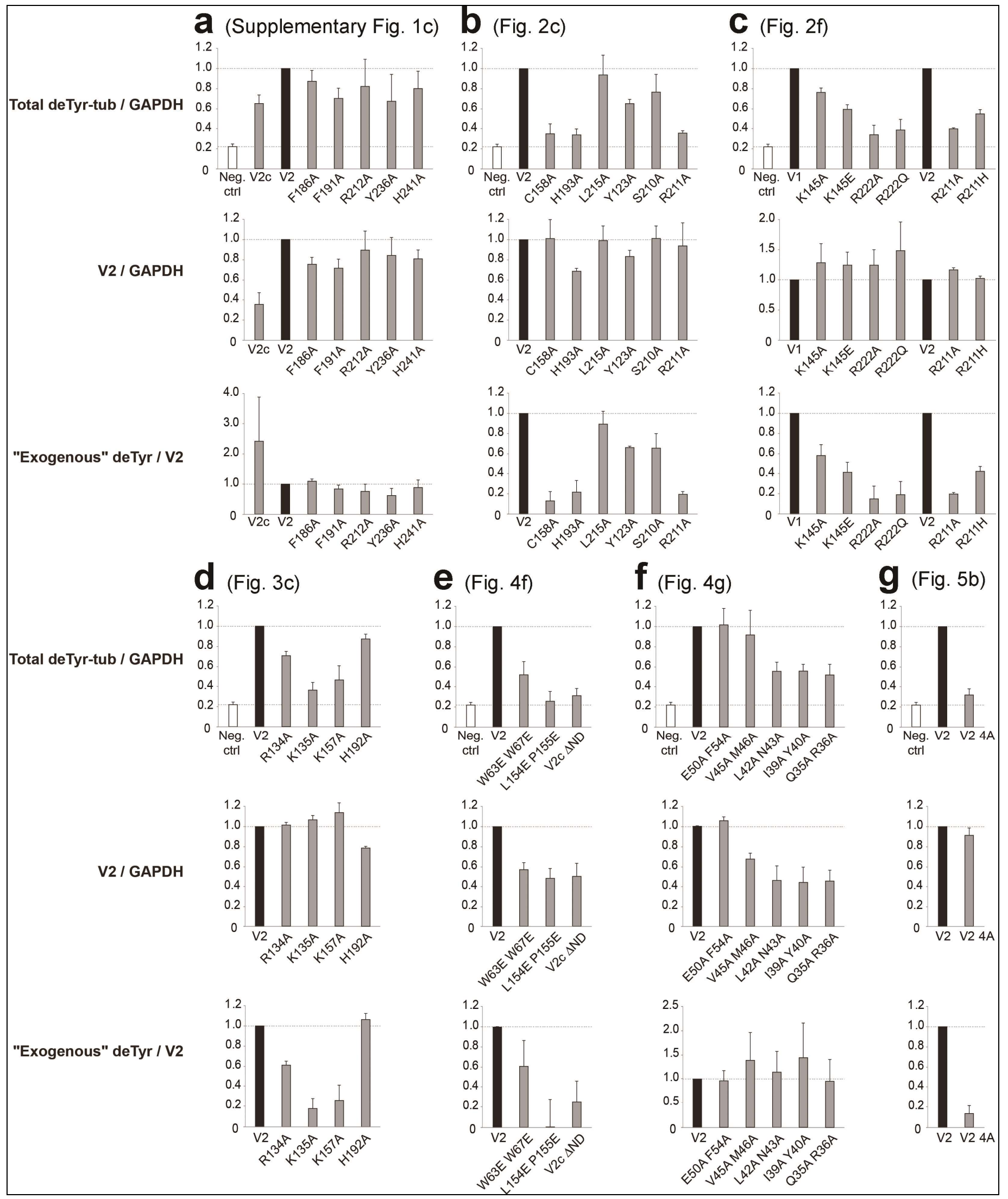




\section{Supplementary Figure 2}

Quantification of tubulin detyrosination assay immunoblots.

Quantification results of immunoblots corresponding to Supplementary Fig. 1c (a) and Figs 2c (b), 2f (c), 3c (d), 4f (e), 4g (f), and 5b (g). Immunoblots, resulting each from an independent transfection, were analyzed for the negative control (sham transfection, white column, $n=16$ total), the wild type non-mutated vasohibins (black columns, $n=3-5$ per graph) or mutated vasohibins (gray columns, $n$ $=3$ except for F186A and Y236A in panel $\mathbf{a}(n=2)$, and W63E W67E in panel $\mathbf{e}(n=4)$ ), and expressed as mean \pm sem. In the top graphs, the plotted values represent the relative intensities of the detyrosinated-tubulin (deTyr-tub) signal obtained with mutants after normalization to the GAPDH signal. The signal of the wild type vasohibin from the same blots was set to 1.0, upper dotted line. Note that this measurement corresponds to endogenous (bottom dotted line) plus exogenous detyrosination activities in cells. In the middle graphs, the plotted values represent the relative quantities of mutant vasohibins expressed in cells after normalization to the GAPDH signal. The signal of the wild type vasohibin from the same blots was set to 1.0 , upper dotted line. Note that this signal only corresponds to exogenous vasohibin levels since detection was performed using an anti-eGFP antibody. In the bottom graphs, the plotted values represent the relative intensities of the deTyr-tubulin signal obtained with mutants after subtracting the HEK293T cells endogenous levels of deTyr-tubulin (obtained with sham transfected cells, white columns in top graphs). The signal obtained with wild type vasohibin from the same blots was set to 1.0, upper dotted line. All The source files are available in Supplementary Data Set 2. 


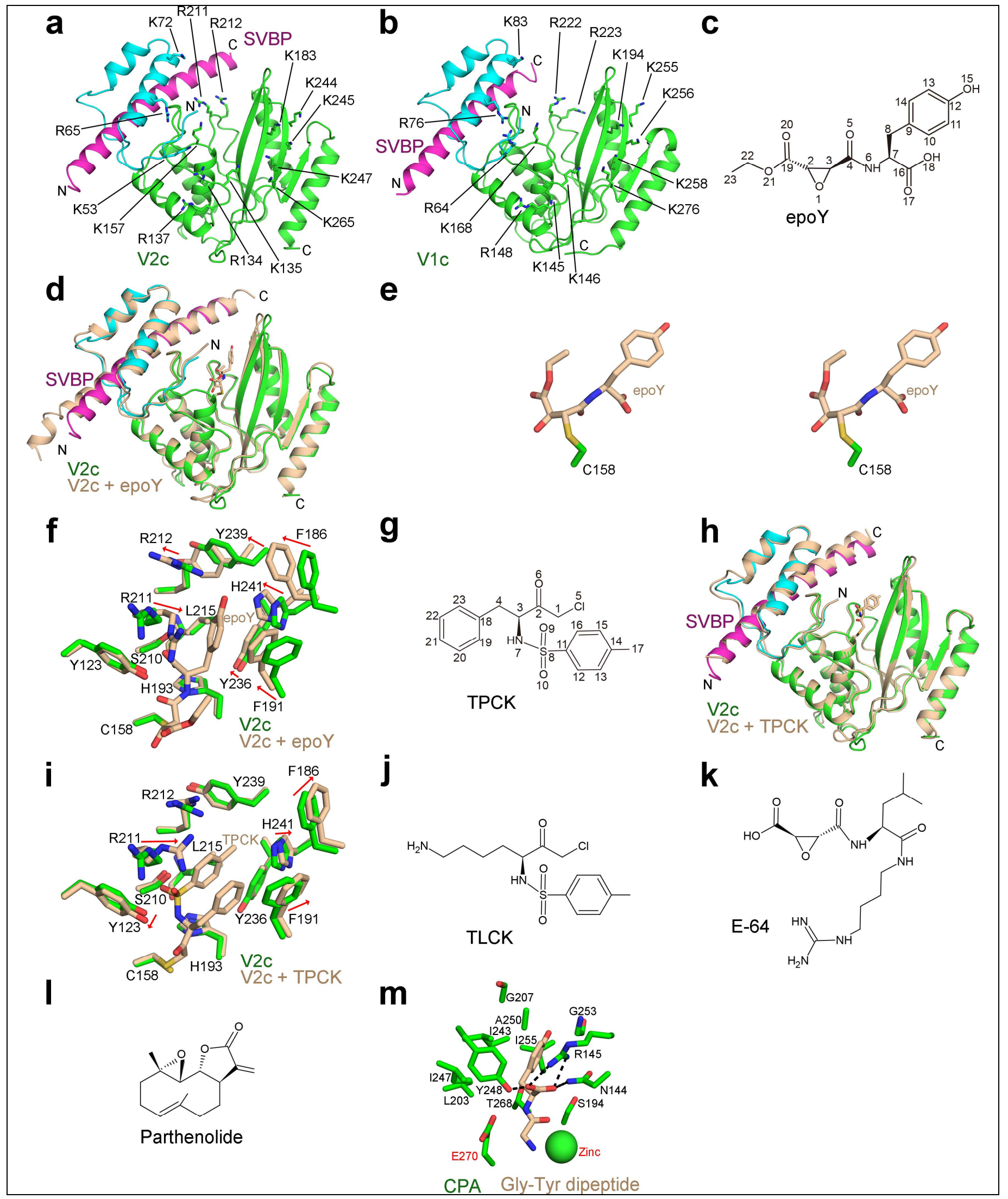


Supplementary Figure 3

Characteristics of the positively charged groove of vasohibins and inhibition of vasohibins by small molecules.

a-b, 14 conserved lysine and arginine residues (see also Supplementary Note) shape the positively charged groove of the V2c-SVBP (a) and V1c-SVBP (b) structures. c, Chemical structure of epoY that inhibits vasohibins with very good potency. $\mathbf{d}$, Superimposition of the structures of V2c-SVBP (color coded as in Fig. 1a) and V2c-SVBP-epoY (wheat) in cartoon representation with the epoY shown in sticks representation. The rmsd of the two superimposed structures is $0.61 \AA$. e, Related to (d), walleye stereo view highlighting the covalent linkage between epoY and the catalytic residue C158 of V2c. f, Superimposition of the active site residues of V2c-SVBP (green) onto the ones of V2c-SVBP-epoY (wheat) in sticks representation. The red arrows highlight the conformational changes that residues experience upon ligand binding. $\mathbf{g}$, Chemical structure of TPCK that inhibits vasohibins with very good potency. $\mathbf{h}$ Superimposition of the structures of V2c-SVBP (color coded as in Fig. 1a) and V2c-SVBP-TPCK (wheat) in cartoon representation with the ligand TPCK shown in sticks. The rmsd is $0.43 \AA$. $\mathbf{i}$, Superimposition of the active site residues of V2c-SVBP (green) onto the ones of V2c-SVBP-TPCK (wheat) in sticks representation. The red arrows highlight the conformational changes that residues experience upon ligand binding. j-I, Chemical structures of TLCK (j), E-64 (k) and parthenolide (I). The ligands TLCK, E-64 and parthenolide only show modest inhibitory activity against vasohibins. $\mathbf{m}$, Zoom in view on the active site residues of the structure of carboxypeptidase A (CPA) in complex with a Gly-Tyr dipeptide (PDB ID 3CPA). Interacting residues of CPA (green) with the Gly-Tyr dipeptide (wheat) are shown in sticks representation. Both the catalytic residue E270 and the catalytic zinc atom are also highlighted with the zinc-coordinating residues omitted for clarity. 


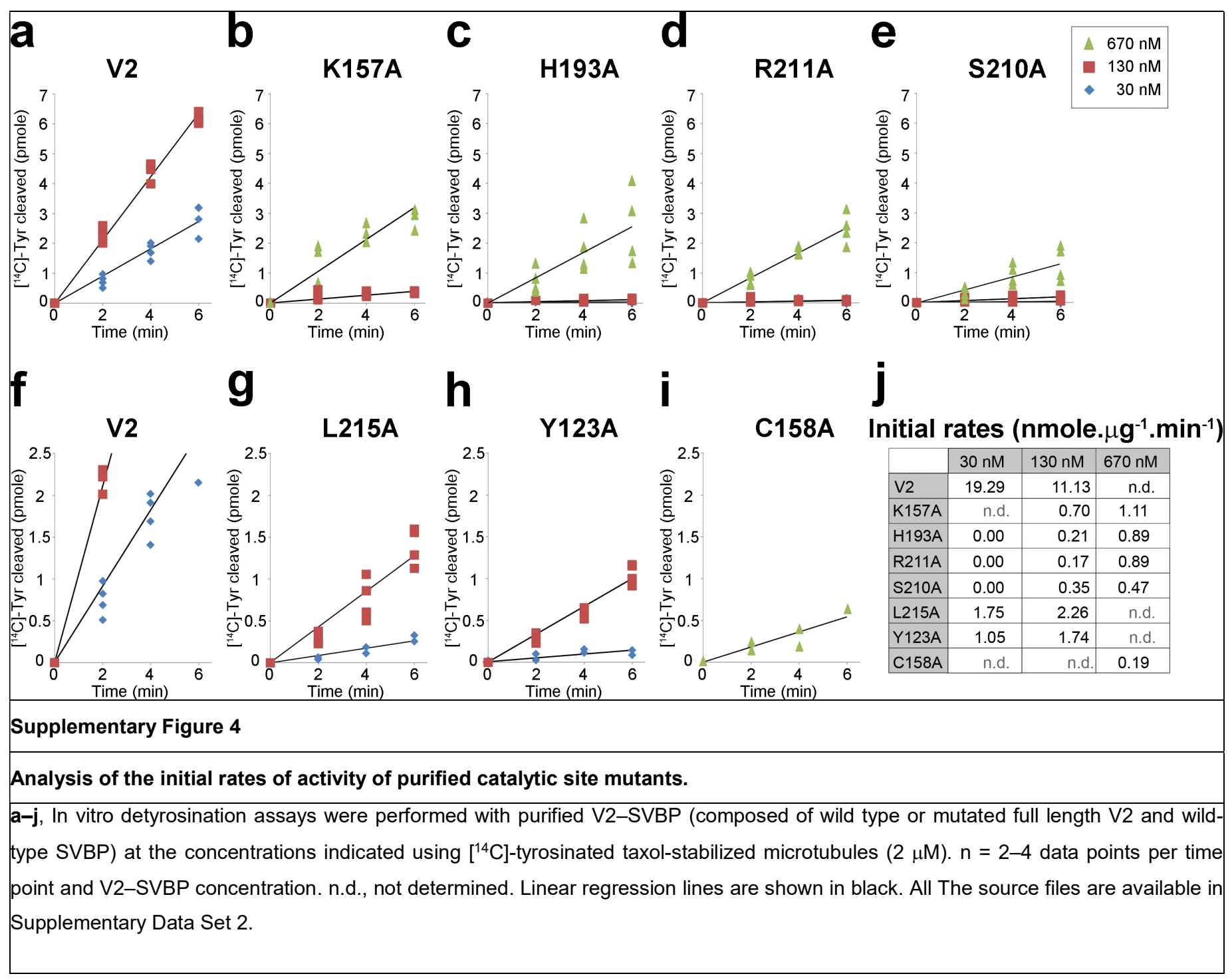




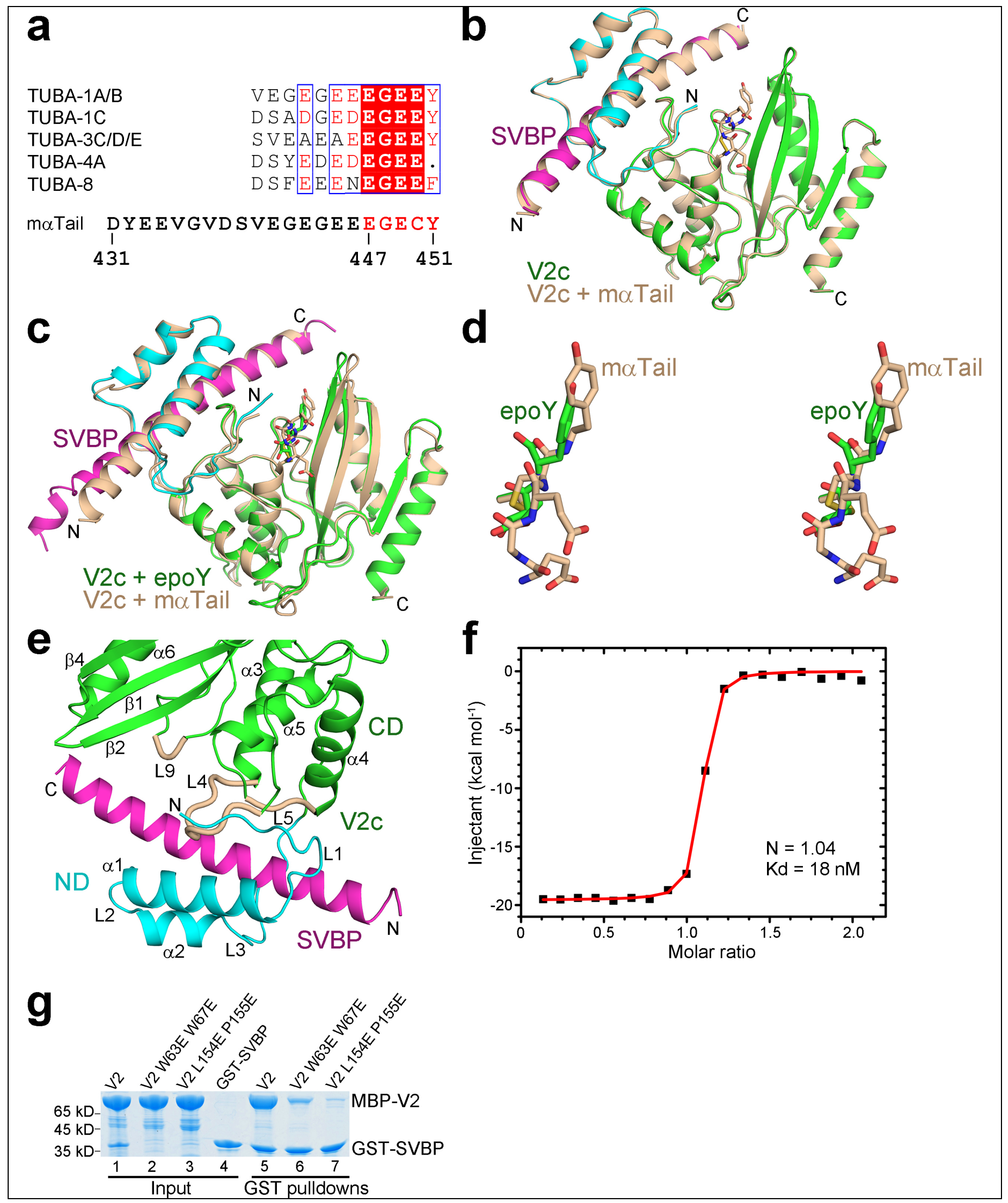




\section{Supplementary Figure 5}

\section{Analysis of the V2c-SVBP-m $\alpha$ Tail structure and characterization of the vasohibin-SVBP interaction.}

a, Sequence alignment of the C-terminal tails of the human $\alpha$-tubulin isotypes. Identical and conserved residues are highlighted. The sequence of the maTail used for crystallization is derived from the TUBA-1A/B isotypes and shown below the alignment. The 447EGECY-451 segment (colored in red) of the maTail is resolved and modeled in the V2c-SVBP-maTail structure, while the other residues of the maTail are disordered in the structure. b. Superimposition of the structures of V2c-SVBP (color coded as in Fig. 1a) and V2c-SVBP-maTail (wheat) in cartoon representations, with the maTail peptide (wheat) shown in sticks representation. The rmsc of these two superimposed structures is $0.19 \AA$. c, Superimposition of the structures of V2c-SVBP-epoY (color coded as in Fig. 1a) and V2c-SVBP-maTail (wheat) in cartoon representations, with the epoY (green) and maTail peptide (wheat) shown in sticks representations. The rmsd of these two superimposed structures is $0.46 \AA$. d, Related to panel (c), walleye stereo view of the ligand epoY (green) and the peptide maTail (wheat) after superimposition of the V2c-SVBP-epoY and V2c-SVBP-maTail structures. e, Overall view of the interacting structural elements between V2c and SVBP in the V2c-SVBP structure in cartoon representation. The SVBP $\alpha$-helix (magenta) is wrapped around by the N-terminal domain (ND; cyan) of V2c and is further contacted by the C-terminal domain (CD; green) of V2c through the three loops L4, L5 and L9 (highlighted with wheat tubules). Detailed interactions between V2c and SVBP are showed in Fig. 4a-c. f, ITC experiment with purified MBP-V2c and SVBP proteins. The source file is available in Supplementary Data Set 2. g, SDS-PAGE of GST pull-down experiments performed with different MBP-V2 mutants and wild type GSTSVBP, (see also Fig. 4d). Both distinct V2 double point mutations impair the interaction with SVBP. Uncropped images are shown in Supplementary Data Set 1. 

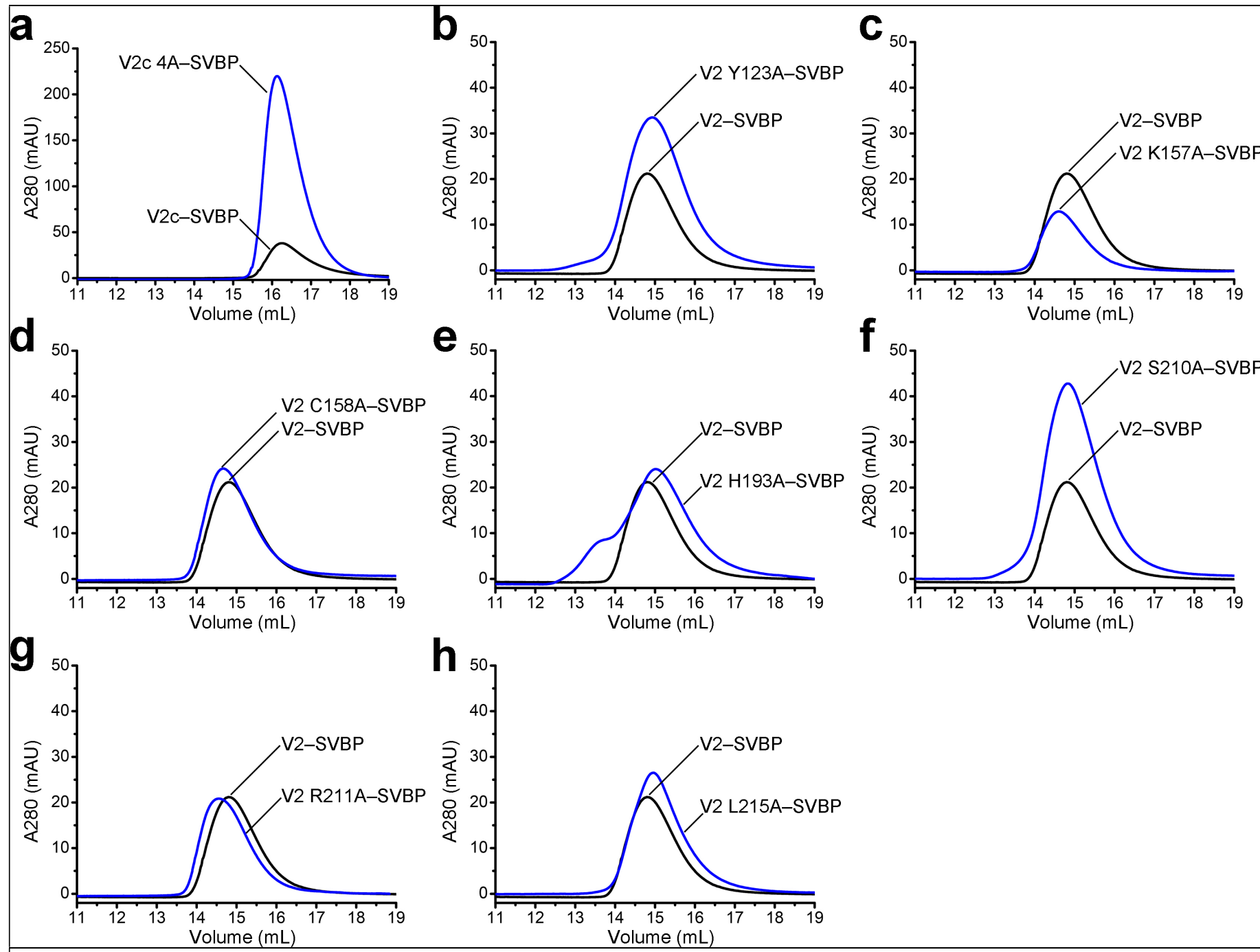

\section{Supplementary Figure 6}

Size exclusion chromatography profiles of mutants.

a, Size exclusion chromatography profile of V2c 4A mutant. The V2c 4A-SVBP complex was compared with the wild type V2c-SVBP complex using size exclusion chromatography. The profile suggests that the V2 4A mutant is indeed folded properly. $\mathbf{b}-\mathbf{h}$, Most of the complex mutants used in this study were also analyzed and compared with the wild type V2-SVBP complexes using size exclusion chromatography. The profiles also suggest that all V2 variants are folded properly. 

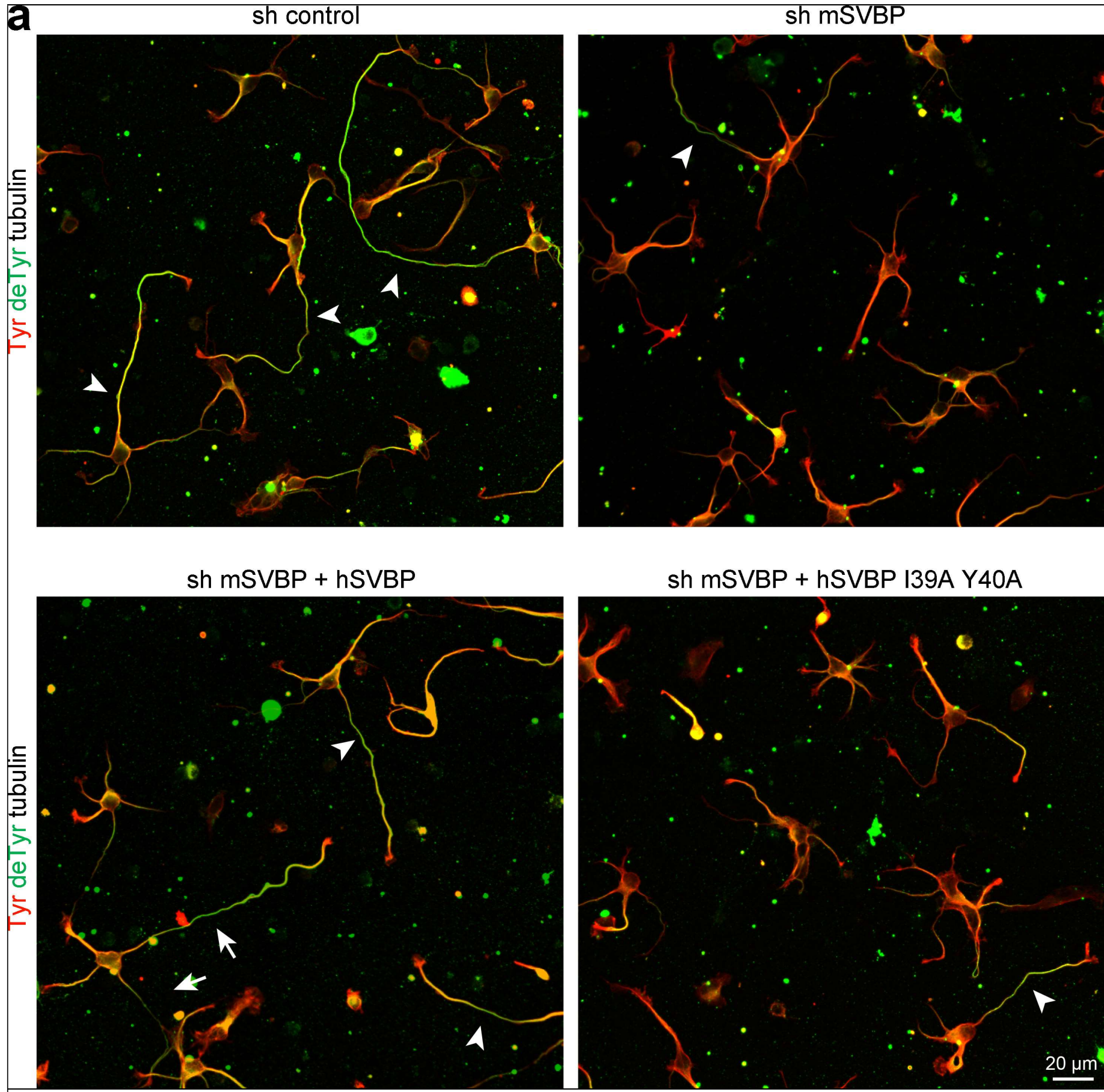

Supplementary Figure 7

The vasohibin-SVBP interaction is crucial for axon specification.

a, Wild type human SVBP (hSVBP), but not its double mutant I39A Y40A, rescues axonal specification in mouse hippocampal neurons knocked down for mouse SVBP (mSVBP). Representative immunofluorescence images of neurons cotransfected with plasmids expressing a shRNA (CTRL or mSVBP) and SVBP-myc-Flag (wild type or I39A Y40A). Tyrosinated and detyrosinated $\alpha$-tubulin contents were assayed at 1 DIV and staining images were merged. Detyrosinated tubulin levels and number of stage III neuron (bearing 
an axon) are significantly reduced in knocked down neurons (top right: sh mSVBP) compared to control neurons (top left: sh control) and rescued with native hSVBP (bottom left: sh mSVBP + hSVBP) but not with the hSVBP I39A Y40A mutant (bottom right: sh mSVBP + hSVBP I39A Y40A). Scale bar is indicated. Arrowheads and arrows highlight single and double axons emanating from a cell body, respectively. 


\section{Supplementary Note}
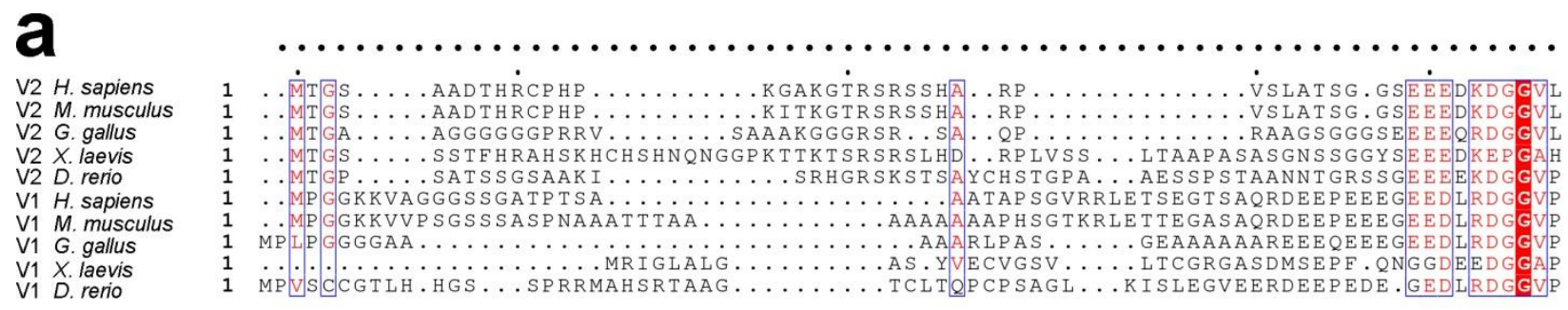

V2 H. sapiens
V2 M. musculus
V2 G. gallus
V2 X. laevis
V2 $D$. rerio
V1 H. sapiens
V1 M. musculus
V1 G. gallus
V1 X. laevis
V1 D. rerio
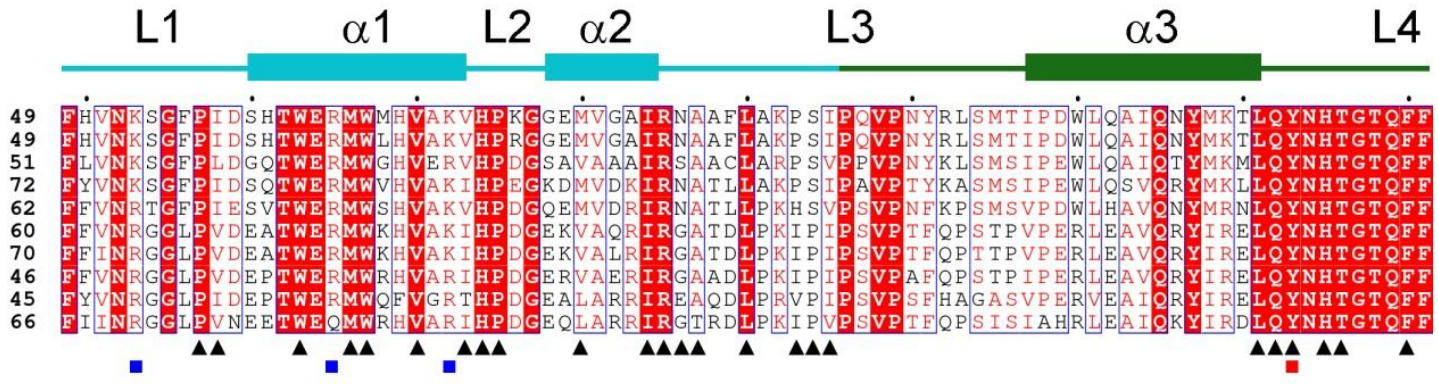

V2 H. sapiens
V2 M. musculus
V2 G. gallus
V2 X. laevis
V2 $D$. rerio
V1 H. sapiens
V1 M. musculus
V1 G. gallus
V1 X. laevis
V1 D. renio

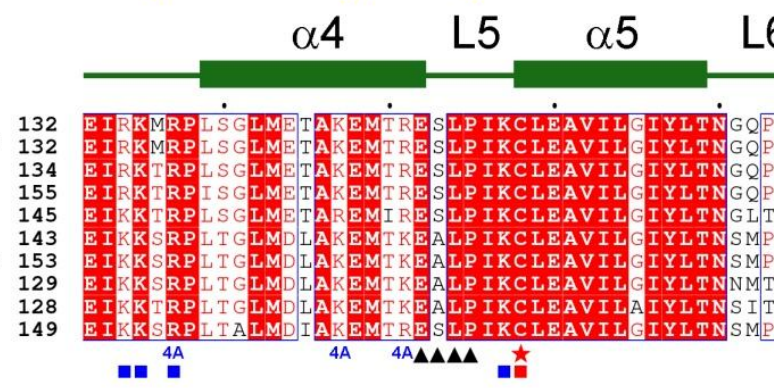

L6 $\quad \beta 1$

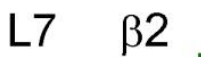

L8 $\beta 3$

L9

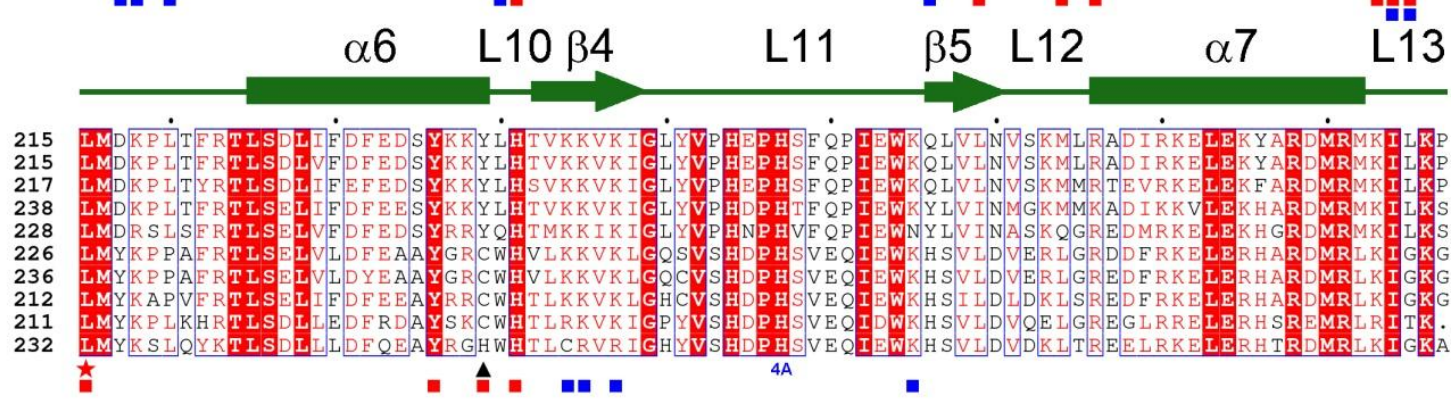

L11

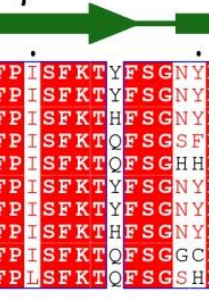

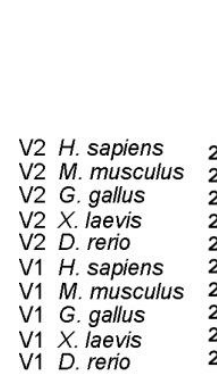

V2 H. sapiens

V2 M. musculus

V2 G. gallus

V2 $X$. laevis

V2 D. rerio

V1 $H$. sapiens

V1 M. musculus

V1 G. gallus

V1 X. laevis

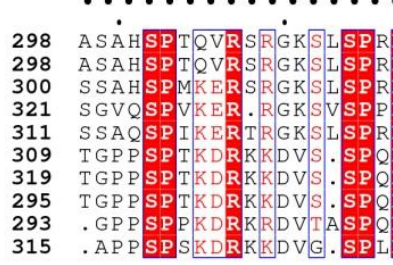

b

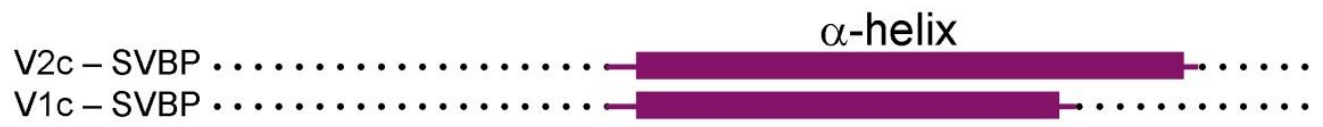

SVBP H. sapiens 1 M்DP. PARKEKTंKVKESVISRV SVBP M. musculus

SVBP G. gallus

SVBP X. laevis

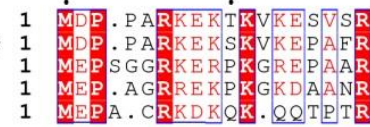

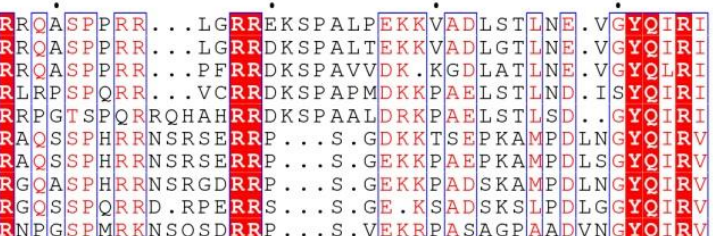

SVBP D. rerio 


\section{Supplementary Note. Multiple sequence alignments of vasohibins and SVBP.}

a, Sequence alignment of V2 and V1 from different species: H. sapiens V2 (NP_001287985) and V1 (NP_055724); M. musculus V2 (NP_659128) and V1 (NP_796328); G. gallus V2 (XP_015139368) and V1 (XP_015143201); X. laevis V2 (XP_018118075) and V1 (XP_018085836); D. rerio V2 (XP_005160797) and V1 (XP_003200451). Identical residues across species are highlighted as white letters on a red background; conserved residues are highlighted with red letters on a white background. Well conserved sequence regions are highlighted by blue boxes. Secondary structure elements derived from the crystal structure of human V2c-SVBP are shown on top of the sequence alignment with the ND and CD domains of V2c colored in cyan and green, respectively. The disordered $\mathrm{N}$ - and C-regions in V2 are indicated by black dots. Under the alignment, red ' $\star$ ' highlights the catalytic triad residues of vasohibins, which are C158, H193 and L215 in human V2; blue ' $\square$ ' highlights the 14 conserved lysine and arginine residues within or surrounding the positively-charged groove of V2c-SVBP; red ' $\mathrm{m}$ ' highlights the 12 conserved residues forming the tyrosine-binding pocket of V2c-SVBP: with Y123, C158, F186, F191, H193, S210, R211, R212, L215, Y236, and H241 being strictly-conserved, and Y239 being conserved; black ' $\boldsymbol{\Delta}$ highlights the residues of V2c interacting with SVBP; ' $4 A$ ' highlights a basic cluster of 4 highly conserved residues $\mathrm{R} 137, \mathrm{~K} 147, \mathrm{R} 151$, and $\mathrm{H} 257$, on the surface of V2c, that potentially interact with microtubules in cellulo.

b, Sequence alignment of SVBP from different species: H. sapiens SVBP (NP_955374); M. musculus SVBP (NP_077782); G. gallus SVBP (XP_015152726); X. laevis SVBP (XP_018083777); D. rerio SVBP (NP_001189361). Identical residues across species are highlighted as white letters on a red background; conserved residues are highlighted with red letters on a white background. Well conserved sequence regions are highlighted by blue boxes. Secondary structure elements of SVBP derived from the V2cSVBP and V1c-SVBP structures are shown on top of the alignment. Residues that were not visible in the

V2c-SVBP structure are indicated by black dots. Under the alignment, black ' $\boldsymbol{\Lambda}$ ' highlights the residues of SVBP interacting with V2c. 